A Model for the Plastic Flow of Landslides

U.S. GEOLOGICAL SURVEY PROFESSIONAL PAPER 1385

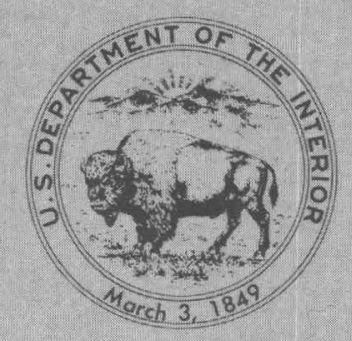





\section{A Model for the Plastic Flow of Landslides}

By WILLIAM Z. SAVAGE and WILLIAM K. SMITH

U.S. GEOLOGICAL SURVEY PROAESIONAL PAPER 1385 An application of the theory of Coulomb plasticity to the flow of landslides

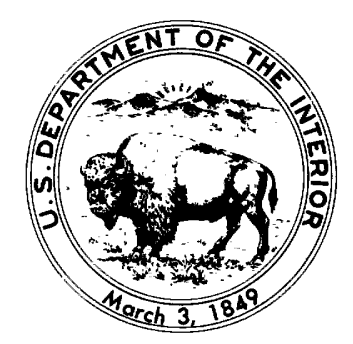




\section{DEPARTMENT OF THE INTERIOR}

DONALD PAUL HODEL, Secretary

\section{U.S. GEOLOGICAL SURVEY}

Dallas L. Peck, Director

Library of Congress Cataloging in Publication Data

Savage, William Z.

A model for the plastic flow of landslides.

(U.S. Geological Survey professional paper ; 1385)

"An application of the theory of Coulomb plasticity to the flow of landslides."

Bibliography: p.

Supt. of Docs. no.: I 19.16:1385

1. Landslides-Mathematical models. 2. Plasticity-Mathematical models. I. Smith William K. II. Title. III. Series: Geological Survey professional paper : 1385.

QE599.A2S28 1986

For sale by the

Books and Open-File Reports Section

U.S. Geological Survey

Federal Center

Box 25425

Denver, CO 80225

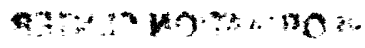




\section{CONTENTS}

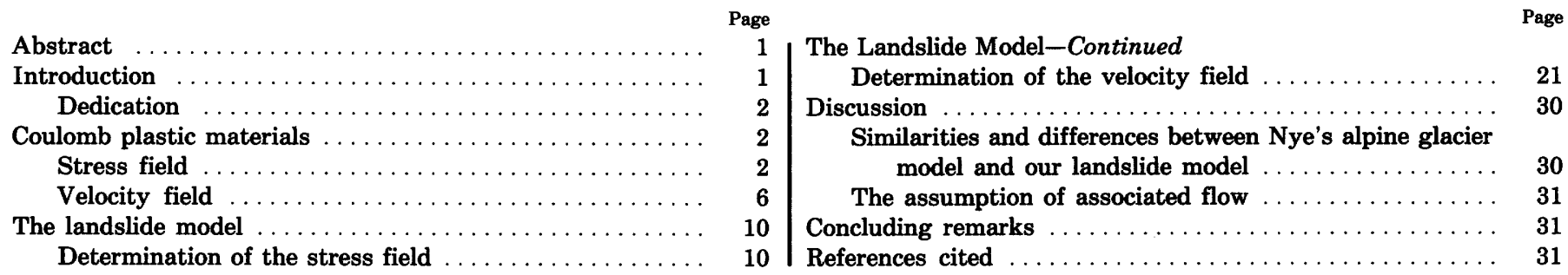

\section{ILLUSTRATIONS}

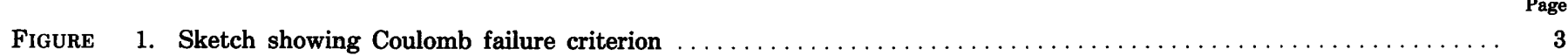

2. Sketch showing Cartesian coordinate system inclined at an angle $\theta$ to the horizontal $\ldots \ldots \ldots \ldots$

3. Sketch showing semi-infinite mass of soil inclined at an angle $\theta$ to the horizontal $\ldots \ldots \ldots \ldots \ldots$

4-13. Graphs showing:

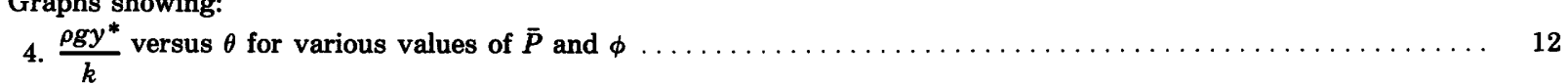

5. Bounds on $\overline{\boldsymbol{P}}$ as a function of slope angle $\theta$ for various angles of internal friction $\phi \ldots \ldots \ldots \ldots$

6. Variation of $\bar{\sigma}_{x}^{\prime}, \bar{\sigma}_{x y}$, and $\bar{\sigma}_{y}^{\prime}$ with $y / y^{*}$ for $\phi=30^{\circ}, \theta=20^{\circ}$, and $\bar{P}=0.5$ in extending and compressive flow $\ldots 15$

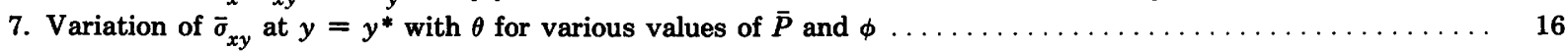

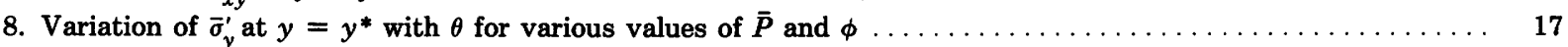

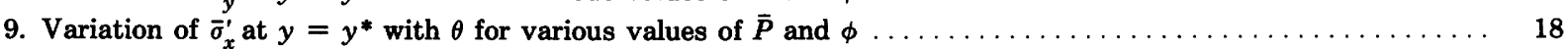

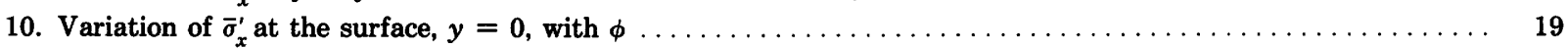

11. Dimensionless depth $y / y^{*}$ to which $\bar{\sigma}_{x}^{\prime}$ is tensile in extending flow as a function of $\theta$ for various values of $\bar{P}$ and $\phi \quad 20$

12. Dimensionless depth $y / y^{*}$ of maximum values of $\bar{\sigma}_{x}^{\prime}$ in compressive flow as a function of $\theta$ for various values of $\phi$

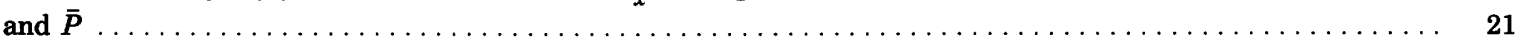

13. Maximum value of $\bar{\sigma}_{x}^{\prime}$ in compressive flow as a function of $\theta$ for various values of $\phi$ and $\bar{P} \ldots \ldots \ldots 22$

14. Sketches showing stress characteristics for various slope angles $\theta$, angles of internal friction $\phi$, and dimensionless pore pressures $\overline{\boldsymbol{P}}$

15. Graphs showing variation of dimensionless dilatancy rate $\dot{d} /|r|$ with dimensionless depth $y / y^{*}$ for various values of

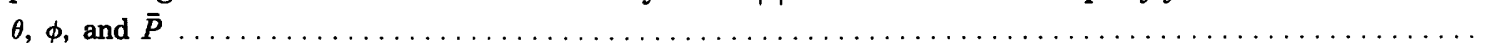

16. Graphs showing dimensionless velocities $\left(u-u_{o}\right) / r y^{*}$ and $v / r y^{*}$ as functions of dimensionless depth $y / y^{*}$ for compressive

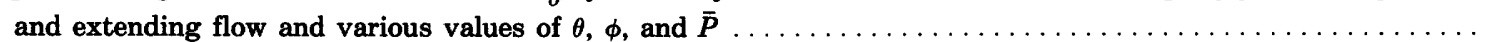

17. Sketches showing fault surfaces produced by propagation of discontinuities along $\beta_{\text {_ }}$ slip lines in $A$, compressive flow

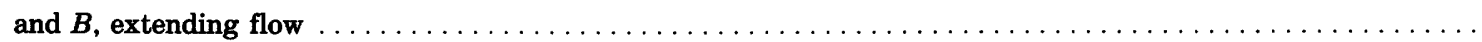

18. Graph showing magnitude of resultant velocity jump on the surface as a function of angle of internal friction $\phi$ for

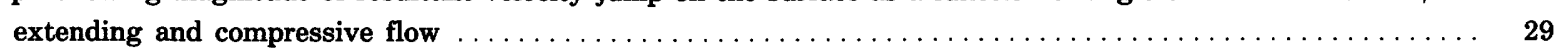

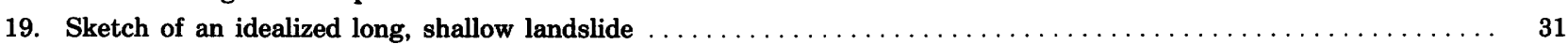




\section{SYMBOLS}

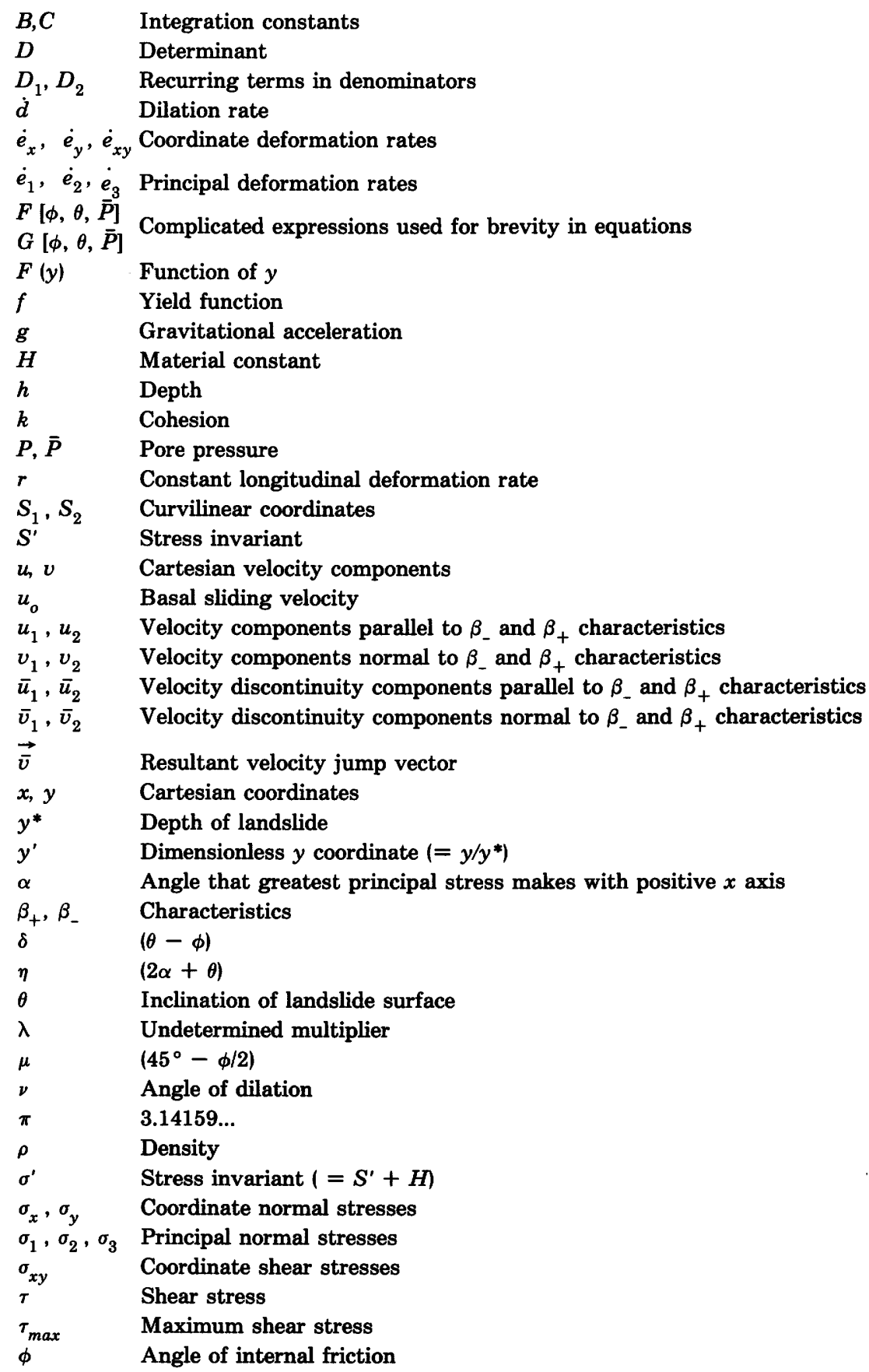

Symbols may be further modified by subscripts or primes. Generally, letter subscripts refer to coordinate directions or directions normal to given planes, whereas numeric subscripts refer to principal directions. Exceptions are $D_{1}, D_{2}, F_{1}[\phi, \theta, \bar{P}], G_{2}[\phi, \theta, \bar{P}]$, etc., where the subscripts merely distinguish between similar expressions. Effective stresses, the difference between total normal stresses and the pore pressure, are denoted by primes. 


\title{
A MODEL FOR THE PLASTIG FLOW OF LANDSLIDES
}

\author{
By William Z. Savage and William K. SMith
}

\begin{abstract}
To further the understanding of the mechanics of landslide flow, we present a model that predicts many of the observed attributes of landslides. The model is based on an integration of the hyperbolic differential equations for stress and velocity fields in a two-dimensional, inclined, semi-infinite half-space of Coulomb plastic material under elevated pore pressure and gravity.

Our landslide model predicts commonly observed features. For example, compressive (passive), plug, or extending (active) flow will occur under appropriate longitudinal strain rates. Also, the model predicts that longitudinal stresses increase elliptically with depth to the basal slide plane, and that stress and velocity characteristics, surfaces along which discontinuities in stress and velocity are propagated, are coincident. Finally, the model shows how thrust and normal faults develop at the landslide surface in compressive and extending flow.
\end{abstract}

\section{INTRODUCTION}

To further the understanding of landslide mechanics we have developed a model that describes many of the observed attributes of landslides. This model, based on the flow theory of Coulomb plastic materials, is similar in many respects to a previous model for alpine glaciers developed by Nye (1951). In this section, we review the principal results of Nye's model and other work that influenced ours, explain how the report is laid out, and give our principal results.

Nye (1951) successfully applied the flow theory of a perfectly plastic incompressible Mises material (Hill, 1950; Varnes, 1962) to the flow of alpine glaciers. His model predicted many of the flow patterns observed in alpine glaciers. For example, he predicted that either active (extending), passive (compressive), or plug flow would occur depending on the longitudinal deformation rate. Nye based his predictions on the assumptions that shear stresses and that vertical normal stresses varied linearly with depth, and found that longitudinal stresses varied elliptically with depth in the glacier. Nye also found that, in active (extending) flow, the longitudinal stresses were tensile, which explains the crevasses commonly observed in parts of glaciers undergoing extending flow. Nye found that the predicted slip-line field explained the thrust surfaces seen in compressiveflow regions of glaciers and normal faults observed in extending-flow regions. For the extending- and compressive-flow states, Nye's model predicted the longitudinal velocity was greatest at the surface and decreased elliptically toward the base. Finally, his model predicted the glacier to simply slide on its base as a rigid body during plug flow.

Landslides have many of the same features as Nye modeled in alpine glaciers. For example, extending, compressive, and plug flows occur, and tensile regions and normal fault scarps develop near the surface in parts of the landslide undergoing extending flow. Also, thrust surfaces and thrust-fault scarps occur in regions of compressive flow. These similarities are not surprising as soils in general behave as materials with plastic states characterized by Coulomb's law, which is a generalization of the Mises criterion for plastic material (Hill, 1950).

Following Nye's lead in modeling the flow of an alpine glacier, we demonstrate that, as with Nye's glacier model, our landslide model predicts that either compressive, extending, or plug flow can occur-depending on the longitudinal deformation rate. The shear stresses and vertical normal stresses vary linearly from the surface to the limiting depth of the landslide's slip-line field, and the longitudinal stress increases in an elliptic manner over the same depth. Both the slope angle, and the density and cohesion of ice, control the thickness of the glacier slip-line field. However, the thickness of the slipline field in a landslide in homogeneous and isotropic soil is controlled by the slope angle, by the pore pressure, and by the density, cohesion, and angle of internal friction of the soil. As in the alpine glacier model, longitudinal stresses near the surface of the landslide will be tensile during extending flow, and the slip-line field predicted for the idealized landslide will explain the thrust surfaces seen in compressive-flow regions of landslides.

Also, as in the glacier model, the landslide model predicts that the longitudinal velocity is greatest at the surface and varies in an elliptic manner down to the basal slip plane of the landslide. Finally, like Nye's glacier-flow model, the landslide model predicts that, during plug flow, the landslide simply slides as a rigid body on its basal slip plane.

Besides Nye's important work, our model for landslide flow will draw heavily on other work. Frontard (1922) 
derived equations for the slip-line fields in extending and compressive flow for a semi-infinite mass of Coulomb material whose surface is inclined at an angle greater than the angle of internal friction. (See Terzaghi, 1943 , p. 40-41.) Sokolovskii (1960, p. 38-40; 1965, p. 77-80) gave similar solutions for soils, as did Johnson (1965) for debris flows. In each of these solutions, the slope angle, cohesion, and angle of internal friction of the soil making up the slide control the depth to which the slip-line field can extend. More recently, Crans and Mandl (1981) applied the theory of plastic flow of Coulomb materials to create a model of the development of growth faults in sediment layers. Like Frontard (1922) and Sokolovskii (1960, 1965), Crans and Mandl (1981) found that the slip-line field was bounded at a finite depth when the slope angle exceeds the angle of internal friction. Crans and Mandl (1981) considered the effect of an elevated pore pressure and found that under certain conditions this pressure led to a bounded slipline field, even when the slope angle was less than the angle of internal friction.

In this report, we first develop the general differential equations for stress and velocity fields in twodimensional Coulomb plastic materials under an elevated pore pressure. Our results show that these partial differential equations are hyperbolic and, in general, integrable by a method that uses special curves in an $x-y$ coordinate plane called characteristics, along which the solution of the partial differential equations is reduced to integration of ordinary differential equations. The characteristics are also surfaces across which first derivatives of stress and velocity may be discontinuous. In general, two separate sets of characteristics may occur: one for velocities and one for stresses. However, for the Coulomb plastic material in our model, the stress and velocity characteristics are coincident. The coincidence of the stress and velocity characteristics, that is, the slip lines, is important because these surfaces represent rupture surfaces along which velocity or deformation rate discontinuities occur in landslides. Finally, we see that in a Coulomb material, discontinuities in the tangential-velocity component across a characteristic will be accompanied by a discontinuity in the velocity component normal to the characteristic.

We then proceed to the landslide model, where, because of the assumptions regarding this model, we find that the governing hyperbolic differential equations can be integrated directly. First, we discuss the stresses and the stress characteristics for the idealized landslide, and we give the stress distribution and configuration of the stress characteristics for various values of pore pressure, slope angle, cohesion, and internal friction in dimensionless form for extending and compressive flow. Second, we derive the velocity field and give the distribution of longitudinal and normal velocities for various values of pore pressure, slope angle, cohesion, and internal friction in dimensionless form for extending and compressive flow. Third, we consider the special case of a purely cohesive material-a Mises material-arriving at a solution similar to Nye's (1951) solution for alpine glacier flow. This solution emphasizes that our model for landslide flow is a more general treatment of the gravitational flow of plastic materials for which Nye's solution for alpine-glacier flow is a special case.

Finally, we compare our model of landslide flow with Nye's (1951) model for glacier flow and discuss the applicability of this landslide model to actual landslides. Here, we emphasize the restrictiveness of the assumptions made in developing the landslide model and the main conclusions derived from the model.

\section{DEDICATION}

This work is dedicated to the memory of George $\mathbf{M}$. Sowers.

\section{COULOMB PLASTIC MATERIALS}

\section{STRESS FIELD}

It is known from experiment that at a point in a soil at incipient shear failure there occur two intersecting planes along which the shear stress, denoted $\tau_{n}$, is given by

$$
\left|\tau_{n}\right|=k+\sigma_{n}^{\prime} \tan \phi,
$$

where $k$ is the cohesion, $\phi$ is the angle of internal friction, and $\sigma_{n}^{\prime}$ is the effective normal stress acting on the planes. (Throughout this report effective stresses, the difference between total normal stresses and the pore pressure, are denoted by primes.) The two intersecting planes are equally inclined at angles of $45^{\circ}-\phi / 2$ to the most compressive principal effective stress, $\sigma_{1}^{\prime}$, and the line of intersection of these two planes is parallel to the intermediate principal effective stress, $\sigma_{2}^{\prime}$. Equation (1) was first suggested by Coulomb (1773) for total stresses and is known as the Coulomb failure criterion.

Equation (1) plots as two straight lines in twodimensional Mohr stress space (Terzaghi, 1943, p. 15-24), as shown in figure 1 . When $\phi=0$, the two straight lines in figure 1 are parallel, and the soil is termed purely cohesive or a Mises material. These lines also separate the Mohr stress space into two parts: a stable part and an unstable part. If a Mohr circle 


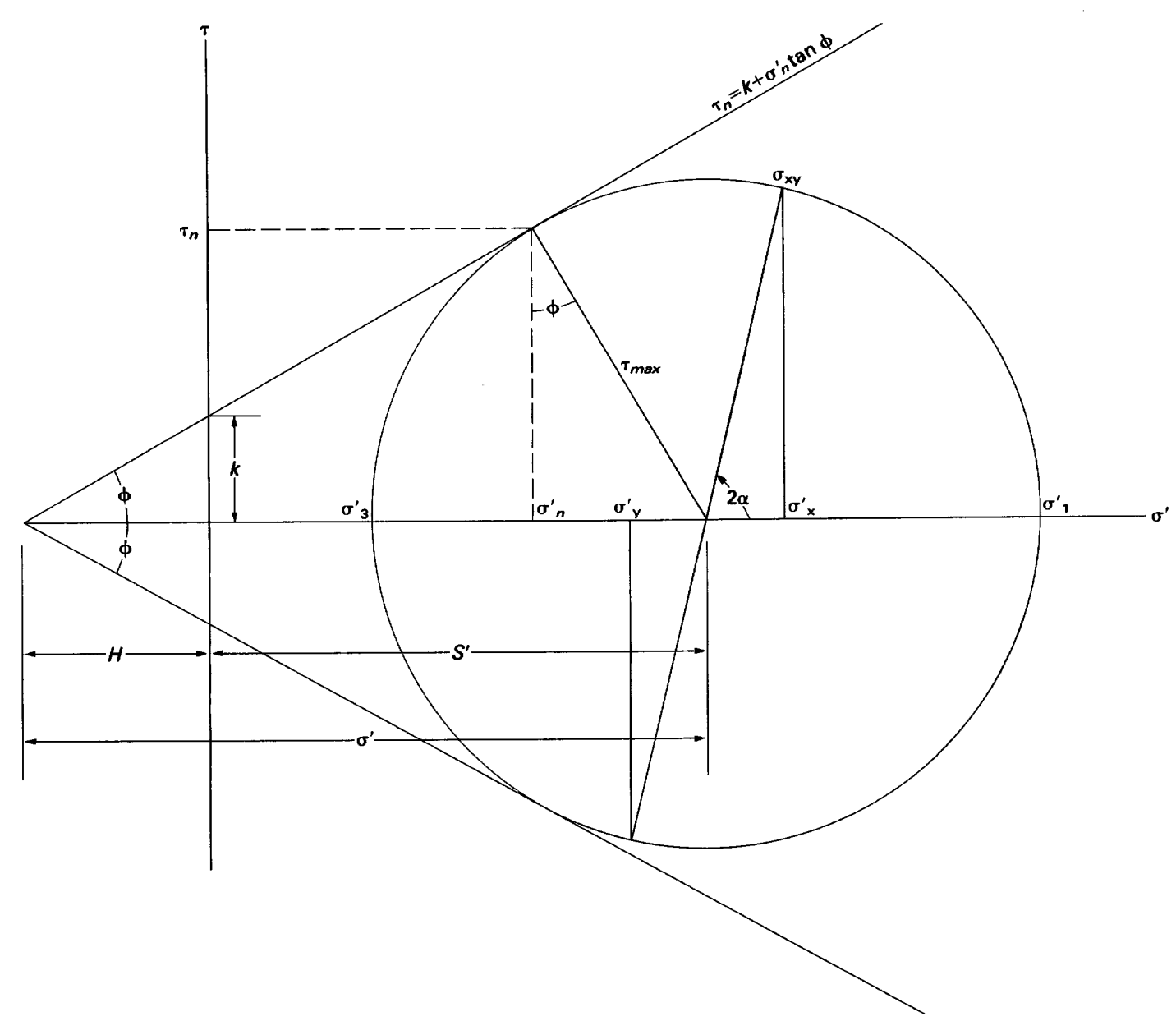

FigURE 1.-Sketch showing Coulomb failure criterion. Normal and shear stresses are represented by $\sigma$ and $\tau$, respectively. Here, $\tau_{\max }$ is the maximum shear stress, $\tau_{n}$ is the shear stress acting on planes inclined at an angle $\alpha$ to $\sigma_{1}^{\prime}$ (the most compressive principal effective stress), $\sigma_{3}^{\prime}$ is the least compressive principal effective stress, $\phi$ is the angle of internal friction, and $k$ is the cohesion. Other symbols are defined in the text.

constructed from $\sigma_{1}^{\prime}$ and $\sigma_{3}^{\prime}$ (the least compressive principal stress at a point) lies between the Coulomb failure lines, the soil behaves elastically. However, if a Mohr circle becomes tangent to these lines, failure is imminent and Coulomb plastic flow will ensue. This state of incipient failure is termed a state of limiting equilibrium. No Mohr circle can lie beyond these limiting lines because the shear stress cannot exceed the yield strength if the body is to remain in equilibrium.

Certain other relationships in a region at limiting equilibrium can be derived from figure 1. For example,

$$
H=k \cot \phi,
$$

and

$$
\tau_{\max }=\frac{\sigma_{1}-\sigma_{3}}{2}=\left(S^{\prime}+H\right) \sin \phi,
$$

where

$$
S^{\prime}=\frac{\sigma_{1}^{\prime}+\sigma_{3}^{\prime}}{2}
$$

Defining

$$
\sigma^{\prime}=S^{\prime}+H
$$

we can write equation (3) as

$$
\tau_{\max }=\sigma^{\prime} \sin \phi .
$$

Also, Coulomb's failure criterion can be written by using equations (1) and (2) as

$$
\left|\tau_{n}\right|=\left(\sigma_{n}^{\prime}+H\right) \tan \phi .
$$

By using the equations for stress transformation 


$$
\begin{aligned}
& \sigma_{x}=\frac{1}{2}\left(\sigma_{1}+\sigma_{3}\right)+\frac{1}{2}\left(\sigma_{1}-\sigma_{3}\right) \cos 2 \alpha, \\
& \sigma_{y}=\frac{1}{2}\left(\sigma_{1}+\sigma_{3}\right)-\frac{1}{2}\left(\sigma_{1}-\sigma_{3}\right) \cos 2 \alpha,
\end{aligned}
$$

and

$$
\sigma_{x y}=\frac{1}{2}\left(\sigma_{1}-\sigma_{3}\right) \sin 2 \alpha,
$$

we can write expressions for the three Cartesian components of stress in a region undergoing Coulomb plastic flow as

$\left.\begin{array}{l}\sigma_{x}^{\prime} \\ \sigma_{y}^{\prime}\end{array}\right\}=S^{\prime} \pm \tau_{\max } \cos 2 \alpha=\sigma^{\prime}(1 \pm \sin \phi \cos 2 \alpha)-H,(9 \mathrm{a})$

and

$$
\sigma_{x y}=\tau_{\max } \sin 2 \alpha=\sigma^{\prime} \sin \phi \sin 2 \alpha .
$$

Here, $\sigma_{x}^{\prime}$ and $\sigma_{y}^{\prime}$ are the normal effective stresses that are positive in compression; $\sigma_{x y}$ is the shear stress; and $\alpha$ is the angle between the direction of the maximum compressive principal effective stress $\sigma_{1}^{\prime}$ and the positive $x$ axis. The magnitudes of $\sigma_{x}^{\prime}, \sigma_{y}^{\prime}$, and $\sigma_{x y}$, as given by equations (8) and (9), are represented in Mohr stress space in figure 1. The angle $\alpha$ is reckoned positive in a counterclockwise direction as shown in figures 1 and 2 . The yield criterion can be written in terms of $\sigma_{x}^{\prime}, \sigma_{y}^{\prime}$, and $\sigma_{x y}$ by using equation (3) or

$$
\tau_{\max }=\frac{\sigma_{1}-\sigma_{3}}{2}=\frac{\left(\sigma_{1}^{\prime}+\sigma_{3}^{\prime}\right)}{2} \sin \phi+H \sin \phi,
$$

and the relations (Sokolovskii, 1965, p. 12)

$$
\frac{\sigma_{1}+\sigma_{3}}{2}=\frac{\sigma_{x}+\sigma_{y}}{2}
$$

and

$$
\frac{\sigma_{1}-\sigma_{3}}{2}=\left[\frac{1}{4}\left(\sigma_{x}-\sigma_{y}\right)^{2}+\sigma_{x y}^{2}\right]^{1 / 2} \text {. }
$$

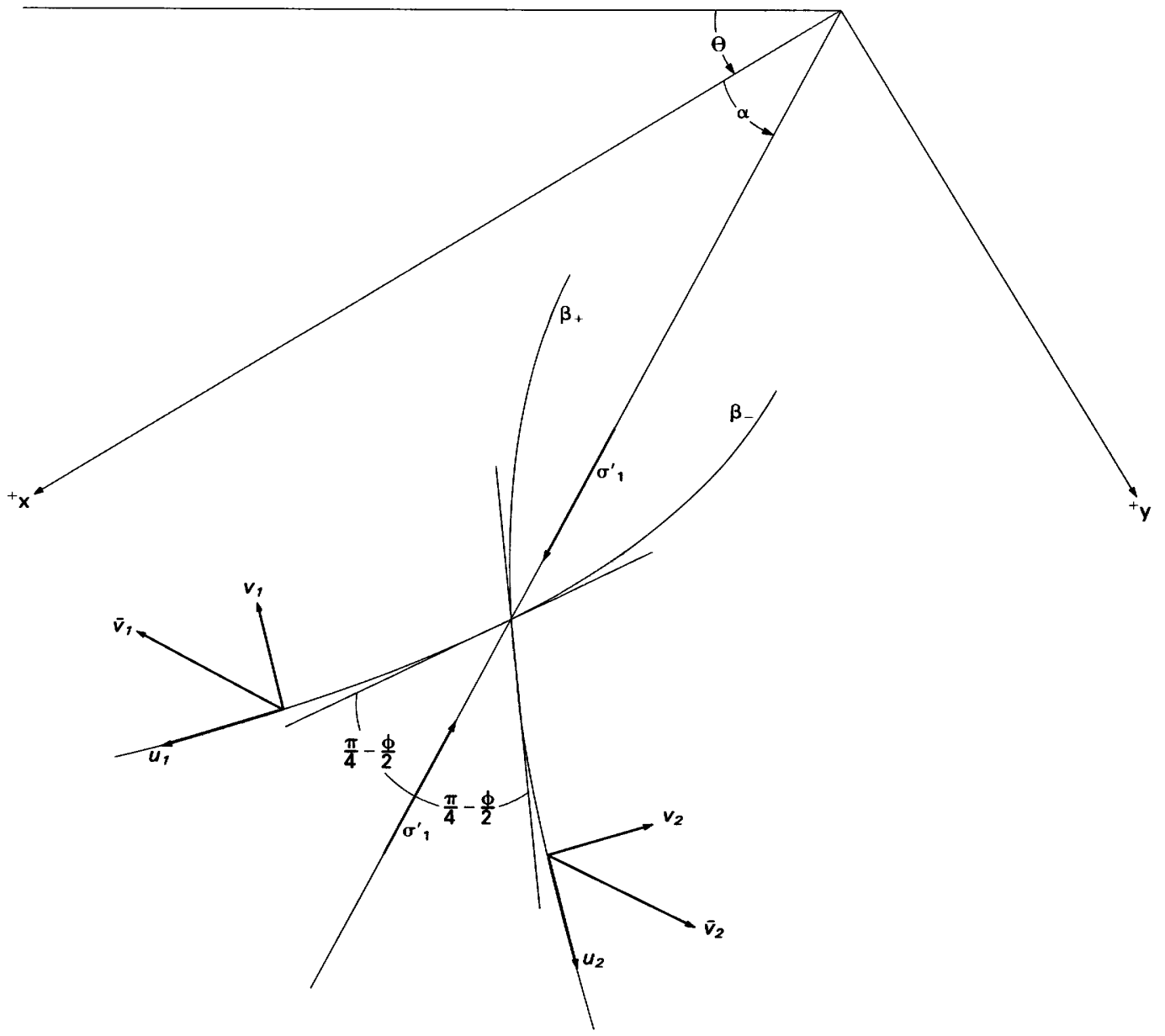

Figure 2.-Sketch showing Cartesian coordinate system inclined at an angle $\theta$ to the horizontal. Also shown are the angle $\alpha$ between the positive $x$ axis and the direction of $\sigma_{1}^{\prime}$; the two stress characteristics $\beta_{+}$and $\beta_{-} ;$the tangents to the two stress characteristics at a point; the velocity components $u_{1}, u_{2}, v_{1}$, and $v_{2}$ on each characteristic; and the resultant velocity vectors $\bar{v}_{1}$ and $\bar{v}_{2}$. 
The yield criterion in terms of $\sigma_{x}^{\prime}, \sigma_{y}^{\prime}$, and $\sigma_{x y}$ then becomes

$$
\frac{1}{4}\left(\sigma_{x}-\sigma_{y}\right)^{2}+\sigma_{x y}^{2}=\frac{\sin ^{2} \phi}{4}\left(\sigma_{x}^{\prime}+\sigma_{y}^{\prime}+2 H\right)^{2} .
$$

In addition to satisfying the yield criterion, stresses in regions of incipient Coulomb plastic flow must also satisfy the differential equations of plane equilibrium

$$
\frac{\partial \sigma_{x}^{\prime}}{\partial x}+\frac{\partial \sigma_{x y}}{\partial y}=\rho g \sin \theta-\frac{\partial P}{\partial x}
$$

and

$$
\frac{\partial \sigma_{x y}}{\partial x}+\frac{\partial \sigma_{y}^{\prime}}{\partial y}=\rho g \cos \theta-\frac{\partial P}{\partial y}
$$

Here, $\rho$ is the density, $\theta$ is the inclination of the $x$ axis from the horizontal (fig. 2 ), and $P$ represents the porewater pressure.

Provided that the pore-pressure distribution is known, the three equations (13), (14a), and (14b) contain three unknowns $\sigma_{x}^{\prime}, \sigma_{y}^{\prime}$, and $\sigma_{x y}$, and can in principle be solved for the stress field if the boundary conditions are expressed in terms of stresses-that is, if the system is statically determinate. The method for solving (13) and $(14 \mathrm{a}, \mathrm{b})$ for the stress field, the method of characteristics, will now be discussed.

Equations $(9 a, b)$, which identically satisfy equation (13), the Coulomb yield condition, are substituted in the equilibrium equations (14a, b), and result in

$$
\begin{gathered}
{[1+\sin \phi \cos 2 \alpha] \frac{\partial \sigma^{\prime}}{\partial x}-2 \sigma^{\prime} \sin \phi \sin 2 \alpha \frac{\partial \alpha}{\partial x}} \\
+\sin \phi \sin 2 \alpha \frac{\partial \sigma^{\prime}}{\partial y}+2 \sigma^{\prime} \sin \phi \cos 2 \alpha \frac{\partial \alpha}{\partial y} \\
=\rho g \sin \theta-\frac{\partial P}{\partial x}
\end{gathered}
$$

and

$\sin \phi \sin 2 \alpha \frac{\partial \sigma^{\prime}}{\partial x}+2 \sigma^{\prime} \sin \phi \cos 2 \alpha \frac{\partial \sigma^{\prime}}{\partial x}$

$$
\begin{gathered}
+[1-\sin \phi \cos 2 \alpha] \frac{\partial \sigma^{\prime}}{\partial y}+2 \sigma^{\prime} \sin \phi \sin 2 \alpha \frac{\partial \sigma^{\prime}}{\partial y} \\
=\rho g \cos \theta-\frac{\partial P}{\partial y} .
\end{gathered}
$$

This result yields two equations for the four derivatives $\frac{\partial \sigma^{\prime}}{\partial x}, \frac{\partial \sigma^{\prime}}{\partial y}, \frac{\partial \alpha}{\partial x}$, and $\frac{\partial \alpha}{\partial y}$. We can add two more equations, the equations of the total derivatives

$$
d \sigma^{\prime}=\frac{\partial \sigma^{\prime}}{\partial x} d x+\frac{\partial \sigma^{\prime}}{\partial y} d y
$$

and

$$
d \alpha=\frac{\partial \alpha}{\partial x} d x+\frac{\partial \alpha}{\partial y} d y,
$$

giving a system of four equations for the four unknown derivatives. In matrix form,

$$
\begin{aligned}
& \left.\begin{array}{cccc}
1+\sin \phi \cos 2 \alpha & \sin \phi \sin 2 \alpha & -2 \sigma^{\prime} \sin \phi \sin 2 \alpha & 2 \sigma^{\prime} \sin \phi \cos 2 \alpha \\
\sin \phi \sin 2 \alpha & 1-\sin \phi \cos 2 \alpha & 2 \sigma^{\prime} \sin \phi \cos 2 \alpha & 2 \sigma^{\prime} \sin \phi \sin 2 \alpha \\
d x & d y & 0 & 0 \\
0 & 0 & d x & d y
\end{array}\right] \\
& \times\left[\begin{array}{c}
\frac{\partial \sigma^{\prime}}{\partial x} \\
\frac{\partial \sigma^{\prime}}{\partial y} \\
\frac{\partial \alpha}{\partial x} \\
\frac{\partial \alpha}{\partial y}
\end{array}\right]=\left[\begin{array}{c}
\rho g \sin \theta-\frac{\partial P}{\partial x} \\
\rho g \cos \theta-\frac{\partial P}{\partial y} \\
d \sigma^{\prime} \\
d \alpha
\end{array}\right] .
\end{aligned}
$$

Following the procedures of matrix algebra, we can invert equation (17) and obtain a unique solution for the four unknown derivatives, provided that the determinant of the square matrix

$$
\begin{gathered}
D=[\cos 2 \alpha+\sin \phi]\left(\frac{d y}{d x}\right)^{2}-2 \sin 2 \alpha \frac{d y}{d x} \\
-[\cos 2 \alpha-\sin \phi]
\end{gathered}
$$

is nonzero. However, if $D=0$, the result is a quadratic form in $\frac{d y}{d x}$ with the two roots

$$
\begin{gathered}
{\left[\frac{d y}{d x}\right]_{ \pm}=\frac{\sin 2 \alpha \pm\left[\sin ^{2} 2 \alpha+\cos ^{2} 2 \alpha-\sin ^{2} \phi\right]^{1 / 2}}{\cos 2 \alpha+\sin \phi}} \\
=\frac{\sin 2 \alpha \pm \cos \phi}{\cos 2 \alpha+\sin \phi}
\end{gathered}
$$




$$
\begin{aligned}
& {\left[\frac{d y}{d x}\right]_{ \pm}=\frac{\sin 2 \alpha \pm \sin \left[90^{\circ}-\phi\right]}{\cos 2 \alpha+\cos \left[90^{\circ}-\phi\right]}} \\
& =\tan \left[\alpha \pm\left(45^{\circ}-\frac{\phi}{2}\right)\right] .
\end{aligned}
$$

The four unknown derivatives, $\frac{\partial \sigma^{\prime}}{\partial x}, \frac{\partial \sigma^{\prime}}{\partial y}, \frac{\partial \alpha}{\partial x}$, and $\frac{\partial \alpha}{\partial y}$, are then no longer unique along two curves in a region of Coulomb plastic flow with tangents at every point given by equation (20). Curves having the slopes $\left[\frac{d y}{d x}\right]_{+}$and $\left[\frac{d y}{d x}\right]_{-}$everywhere are called characteristics. And the partial differential equations (15a, b) are called hyperbolic (Crandall, 1956, p. 354) because there are two real characteristics at a given point. The stress characteristics labeled $\beta_{+}$and $\beta_{-}$are shown in figure 2 .

The stress characteristics intersect at every point at the angle $90^{\circ}-\phi$ (fig. 2) and are loci along which the shear stress is given by equation (1). For a plastically deforming soil, the characteristics represent the potential paths for propagation of discontinuities in stresses and stress gradients. Also, from equation (20), we see that when $\alpha=45^{\circ}-\phi / 2$, the slopes $\left[\frac{d y}{d x}\right]_{ \pm}$are either infinite or zero. Lines of discontinuity, lines along which $\left[\frac{d y}{d x}\right]_{ \pm}$are either infinite or zero, represent boundaries between plastic regions or between plastic and nonplastic regions. Stresses can undergo finite jumps, and stress gradients can be infinite across lines of discontinuity. (See, for example, Prager and Hodge, 1951.) Finally, lines of discontinuity may form on stress characteristics or form an envelope around a system of stress characteristics (Sokolovskii, 1960, 1965).

In addition, for a compatible system of hyperbolic differential equations, the determinant formed by replacing any column on the left of equation (17) with the column on the right must also vanish (Abbott, 1966). For example,

$$
\begin{aligned}
& D= \mid \begin{array}{cccc}
1+\sin \phi \cos 2 \alpha & \sin \phi \sin 2 \alpha & -2 \sigma^{\prime} \sin \phi \sin 2 \alpha & \rho g \sin \theta-\frac{\partial P}{\partial x} \\
\sin \phi \sin 2 \alpha & 1-\sin \phi \cos 2 \alpha & 2 \sigma^{\prime} \sin \phi \cos 2 \alpha & \rho g \cos \theta-\frac{\partial P}{\partial y} \\
d x & \begin{array}{c}
d y \\
0
\end{array} & 0 & d \sigma^{\prime} \\
d x
\end{array} \\
&=\cos ^{2} \phi d \sigma^{\prime}+2 \sigma^{\prime} \sin \phi[(\cos 2 \alpha+\sin \phi) \\
&\left.\left(\frac{d y}{d x}\right)-\sin 2 \alpha\right] d \alpha
\end{aligned}
$$

$$
\begin{gathered}
(1+\sin \phi \cos 2 \alpha)]\left(\frac{d y}{d x}\right) \\
-\left[\left(\rho g \sin \theta-\frac{\partial P}{\partial x}\right)(1-\sin \phi \cos 2 \alpha)-\mid \rho g \cos \theta-\frac{\partial P}{\partial y}\right) \\
\sin \phi \sin 2 \alpha] d x=0 .
\end{gathered}
$$

Substituting $\left[\frac{d y}{d x}\right]_{ \pm}$given by equation (20) in equation (21), we find

$$
\begin{gathered}
d \sigma^{\prime} \pm 2 \sigma^{\prime} \tan \phi d \alpha= \\
\frac{1}{\cos ^{2} \phi}\left[\left(\rho g \sin \theta-\frac{\partial P}{\partial x}\right)(1-\sin \phi \cos 2 \alpha)\right. \\
\left.-\left(\rho g \cos \theta-\frac{\partial P}{\partial y}\right) \sin \phi \sin 2 \alpha\right] d x \\
-\frac{1}{\cos ^{2} \phi}\left[\left(\rho g \sin \theta-\frac{\partial P}{\partial x}\right) \quad(\sin \phi \sin 2 \alpha)\right. \\
\left.-\left(\rho g \cos \theta-\frac{\partial P}{\partial y}\right)(1+\sin \phi \cos 2 \alpha)\right] d y .
\end{gathered}
$$

If the pore-pressure distribution is known, equations (20) and (22) represent a pair of ordinary differential equations for $\alpha$ and $\sigma^{\prime}$ along each characteristic. Presuming that they can be solved for $\sigma^{\prime}$ and $\alpha$, we find the stresses $\sigma_{x}^{\prime}, \sigma_{y}^{\prime}$, and $\sigma_{x y}$ along each characteristic from equations $(9 \mathrm{a}, \mathrm{b})$. In general, this system must be solved numerically (Sokolovskii, 1960, 1965).

\section{VELOCITY FIELD}

Let us adopt the hypothesis of associated plasticity (Hill, 1950; Drucker and Prager, 1952; Shield, 1955) which states that the principal plastic-deformation rates $\dot{e}_{1}, \dot{e}_{2}$, and $\dot{e}_{3}$ are proportional to the gradients of the yield function with respect to the principal stresses $\sigma_{1}^{\prime}$, $\sigma_{2}^{\prime}$, and $\sigma_{3}^{\prime}$. Although the associated flow-rule hypothesis is adopted for mathematical simplicity, it leads to unrealistically large dilation rates. (More will be said about this problem later.)

Writing the yield function (equation 10) as

$f=\frac{1}{2}\left(\sigma_{1}^{\prime}-\sigma_{3}^{\prime}\right)-\frac{1}{2}\left(\sigma_{1}^{\prime}+\sigma_{3}^{\prime}\right) \sin \phi-H \sin \phi=0$,

and taking the appropriate derivatives, we find that

$$
\dot{e}_{1}=\lambda \frac{\partial f}{\partial \sigma_{1}^{\prime}}=\frac{\lambda}{2}(1-\sin \phi),
$$




$$
\dot{e}_{2}=0 \text {, }
$$

and

$$
\dot{e}_{3}=\lambda \frac{\partial f}{\partial \sigma_{3}^{\prime}}=\frac{-\lambda}{2}(1-\sin \phi),
$$

where $\lambda$ is an undetermined parameter that is eliminated in the following equations.

Equation (24b) is a result of assuming that flow occurs only in the plane of $\sigma_{1}$ and $\sigma_{3}$. Adding (24a) and $(24 \mathrm{c})$, we find the mean deformation rate

$$
\frac{\dot{e}_{1}+\dot{e}_{3}}{2}=\frac{-\lambda}{2} \sin \phi \text {. }
$$

Subtracting (24a) from (24c) yields the maximum shear deformation rate

$$
\frac{\dot{e}_{1}-\dot{e}_{3}}{2}=\frac{\lambda}{2} .
$$

Eliminating $\lambda$ from equations (25) and (26) yields the equation of continuity

$$
\dot{e}_{1}+\dot{e}_{3}+\left(\dot{e}_{1}-\dot{e}_{3}\right) \sin \phi=0 .
$$

When $\phi$ is greater than zero, the volume increases during flow. When $\phi=0$, equation (27) becomes the equation of continuity for plane incompressible flow.

We also assume isotropy-that the principal axes of deformation rate and stress coincide. Then, from stress transformation equations $(8 a, b, c)$, we have

$$
\frac{2 \sigma_{x y}}{\sigma_{x}^{\prime}-\sigma_{y}^{\prime}}=\frac{2 \dot{e}_{x y}}{\dot{e}_{x}-\dot{e}_{y}}=\tan 2 \alpha .
$$

By taking $u$ and $v$ to be the velocity components in the $x$ and $y$ directions, respectively, and by taking contracting deformation rates as positive, we can define the deformation rates by

$$
\begin{aligned}
& \dot{e}_{x}=\frac{-\partial u}{\partial x}, \\
& \dot{e}_{y}=\frac{-\partial v}{\partial y},
\end{aligned}
$$

and

$$
\dot{e}_{x y}=-\frac{1}{2}\left(\frac{\partial v}{\partial x}+\frac{\partial u}{\partial y}\right)
$$

Then, with these equations, the condition of isotropy (equation 28) becomes

$$
\frac{\frac{\partial v}{\partial x}+\frac{\partial u}{\partial y}}{\frac{\partial u}{\partial x}-\frac{\partial v}{\partial y}}=\tan 2 \alpha .
$$

Using the deformation-rate transformation equations,

$$
\begin{aligned}
& \dot{e}_{x}=\left[\frac{\dot{e}_{1}+\dot{e}_{3}}{2}\right]+\left[\frac{\dot{e}_{1}-\dot{e}_{3}}{2}\right] \cos 2 \alpha, \\
& \dot{e}_{y}=\left[\frac{\dot{e}_{1}+\dot{e}_{3}}{2}\right]-\left[\frac{\dot{e}_{1}-\dot{e}_{3}}{2}\right] \cos 2 \alpha,
\end{aligned}
$$

and

$$
\dot{e}_{x y}=\left[\frac{\dot{e}_{1}-\dot{e}_{3}}{2}\right] \sin 2 \alpha,
$$

which are of the same form as equations $(8 a, b, c)$ for stress transformation, and equations (25) and (26), we arrive at

$$
\begin{aligned}
& \dot{e}_{x}=\frac{-\partial u}{\partial x}=\frac{-\lambda}{2}[\sin \phi-\cos 2 \alpha], \\
& \dot{e}_{y}=\frac{-\partial v}{\partial y}=\frac{-\lambda}{2}[\sin \phi+\cos 2 \alpha],
\end{aligned}
$$

and

$$
\dot{e}_{x y}=-\frac{1}{2}\left[\frac{\partial v}{\partial x}+\frac{\partial u}{\partial y}\right]=\frac{\lambda}{2} \sin 2 \alpha .
$$

As with equation (11) for the stresses, the sum of the principal deformation rates is an invariant, or

$$
\dot{e}_{1}+\dot{e}_{3}=\dot{e}_{x}+\dot{e}_{y}=-\frac{\partial u}{\partial x}-\frac{\partial v}{\partial y} .
$$

The difference of principal deformation rates from equations $(31 a, b)$ is

$$
\dot{e}_{1}-\dot{e}_{3}=\frac{\dot{e}_{x}-\dot{e}_{y}}{\cos 2 \alpha}=\frac{-\left(\frac{\partial u}{\partial x}-\frac{\partial v}{\partial y}\right)}{\cos 2 \alpha}
$$

We can rewrite equation (27), the equation of continuity, as

$$
-\frac{\partial u}{\partial x}-\frac{\partial v}{\partial y}-\left\lceil\frac{\frac{\partial u}{\partial x}-\frac{\partial v}{\partial y}}{\cos 2 \alpha}\right\rceil \sin \phi=0,
$$


or

$\frac{\partial u}{\partial x}(\cos 2 \alpha+\sin \phi)+\frac{\partial v}{\partial y}(\cos 2 \alpha-\sin \phi)=0$.

Equation (28), the condition of isotropy, is rewritten as

$$
\frac{\partial u}{\partial x} \tan 2 \alpha-\frac{\partial u}{\partial y}-\frac{\partial v}{\partial x}-\frac{\partial v}{\partial y} \tan 2 \alpha=0 .
$$

Equations (36) and (37), together with the equations for the total derivatives

$$
d u=\frac{\partial u}{\partial x} d x+\frac{\partial u}{\partial y} d y
$$

and

$$
d v=\frac{\partial v}{\partial x} d x+\frac{\partial v}{\partial y} d y
$$

yield a system of four equations for the four derivatives $\frac{\partial u}{\partial x}, \frac{\partial u}{\partial y}, \frac{\partial v}{\partial x}$, and $\frac{\partial v}{\partial y}$, or

$\left[\begin{array}{cccc}\cos 2 \alpha+\sin \phi & 0 & 0 & \cos 2 \alpha-\sin \phi \\ \tan 2 \alpha & -1 & -1 & -\tan 2 \alpha \\ d x & d y & 0 & 0 \\ 0 & 0 & d x & d y\end{array}\right]\left[\begin{array}{c}\frac{\partial u}{\partial x} \\ \frac{\partial u}{\partial y} \\ \frac{\partial v}{\partial x} \\ \frac{\partial v}{\partial y}\end{array}\right]=\left[\begin{array}{c}0 \\ 0 \\ d u \\ d v\end{array}\right]$

The determinant of the square matrix is given by the quadratic form

$$
\begin{gathered}
D=(\cos 2 \alpha+\sin \phi)\left(\frac{d y}{d x}\right)^{2}-2 \sin 2 \alpha \frac{d y}{d x} \\
-(\cos 2 \alpha-\sin \phi) .
\end{gathered}
$$

Then, following the procedure outlined for determining the stress characteristics, we find the velocity characteristics

$$
\begin{gathered}
{\left[\frac{d y}{d x}\right]_{ \pm}=\frac{\sin 2 \alpha \pm\left[\sin ^{2} 2 \alpha+\cos ^{2} 2 \alpha-\sin ^{2} \phi\right]^{1 / 2}}{\cos 2 \alpha+\sin \phi}} \\
+\frac{\sin 2 \alpha \pm \cos \phi}{\cos 2 \alpha+\sin \phi}
\end{gathered}
$$

$$
\begin{aligned}
{\left[\frac{d y}{d x}\right]_{ \pm} } & =\frac{\sin 2 \alpha \pm \sin \left[90^{\circ}-\phi\right]}{\cos 2 \alpha+\cos \left[90^{\circ}-\phi\right]} \\
& =\tan \left[\alpha \pm\left(45^{\circ}-\frac{\phi}{2}\right)\right] .
\end{aligned}
$$

Comparing equations (20) and (42), we see that the stress and velocity characteristics coincide. For a plastically deforming soil, the characteristics then represent potential paths for propagating discontinuities in velocity and deformation rates (equations $32 \mathrm{a}, \mathrm{b}, \mathrm{c}$ ), as well as stresses and stress gradients. Because these coincident surfaces (the stress and velocity characteristics) are potential paths for propagating velocity discontinuities, they are called slip surfaces. Like stresses, velocities can also undergo finite jumps and shear- and normal-deformation rates can be infinite across lines of discontinuity - that is, at boundaries between two plastic regions or between plastic and nonplastic regions. Finally, lines of discontinuity can form along velocity characteristics or form an envelope to a system of velocity characteristics.

Like stresses, a compatible system of hyperbolic equations for the velocities requires that the determinant formed by replacing the last column of the square matrix in equation (39) by the column on the right of the equals sign must also vanish. Carrying out this operation, we find that

$$
\begin{aligned}
D & =\left|\begin{array}{crrr}
\cos 2 \alpha \sin \phi & 0 & 0 & 0 \\
\tan 2 \alpha & -1 & -1 & 0 \\
d x & d y & 0 & d u \\
0 & 0 & d x & d y
\end{array}\right| \\
& =d u d x+d v d y=d u+d v \frac{d y}{d x}=0
\end{aligned}
$$

Substituting equations (42) in (43) yields the differential relations between $d u$ and $d v$ along the characteristics $\beta_{+}$and $\beta_{-}$

$$
d u+\tan \left[\alpha \pm\left(45^{\circ}-\frac{\phi}{2}\right)\right] d v=0 .
$$

Here, $\alpha$ is known from the stress solution.

To interpret equation (44), we rewrite it in the canonical forms,

$$
\frac{\partial u}{\partial S_{1}}+\tan [\alpha-\mu] \frac{\partial v}{\partial S_{1}}=0
$$

or

and 


$$
\frac{\partial u}{\partial S_{2}}+\tan [\alpha+\mu] \frac{\partial v}{\partial S_{2}}=0,
$$

where $S_{1}$ is a curvilinear coordinate coincident with the $\beta_{-}$characteristic, $S_{2}$ is a curvilinear coordinate along the $\beta_{+}$characteristic, and $\mu=45^{\circ}-\frac{\phi}{2}$. Next, we define the velocity components $u_{1}$ and $u_{2}$, which are the velocities respectively parallel to the $\beta_{-}$and $\beta_{+}$ characteristics. The relationships between these velocities and the Cartesian velocity components $u$ and $v$ are given by

$$
u_{1}=u \cos (\alpha-\mu)+v \sin (\alpha-\mu)
$$

and

$$
u_{2}=u \cos (\alpha+\mu)+v \sin (\alpha+\mu),
$$

which have the inverses

$$
u=\frac{u_{1} \sin (\alpha+\mu)-u_{2} \sin (\alpha-\mu)}{\cos \phi}
$$

and

$$
v=\frac{u_{1} \cos (\alpha+\mu)+u_{2} \cos (\alpha-\mu)}{\cos \phi} .
$$

Substituting equations $(47 a, b)$ in $(45 a, b)$, we find the canonical equations describing the variation of $u_{1}$ and $u_{2}$ along the $S_{1}$ and $S_{2}$ curvilinear coordinates, the $\beta_{-}$ and $\beta_{+}$characteristics:

$$
\frac{\partial u_{1}}{\partial S_{1}}+\left(u_{1} \tan \phi-\frac{u_{2}}{\cos \phi}\right) \frac{\partial \alpha}{\partial S_{1}}=0
$$

and

$$
\frac{\partial u_{2}}{\partial S_{2}}-\left(u_{2} \tan \phi-\frac{u_{1}}{\cos \phi}\right) \frac{\partial \alpha}{\partial S_{2}}=0 .
$$

Equations $(48 a, b)$ represent the extension rates along the two characteristics (Salencon, 1977) and show that the extension rate along each characteristic vanishes. In addition, we can write equations $(48 a, b)$ as the ordinary differential equations

$$
\frac{d u_{1}}{d \alpha}+\left(u_{1} \tan \phi-\frac{u_{2}}{\cos \phi}\right)=0
$$

and

$$
\frac{d u_{2}}{d \alpha}-\left(u_{2} \tan \phi-\frac{u_{1}}{\cos \phi}\right)=0 .
$$

Equations (48a, b) become the Geiringer equations of perfect plasticity when $\phi=0$ (Davis, 1968, p. 367).

Suppose that a discontinuity in velocity occurs across a $\beta_{-}$characteristic, with the sense of relative displacement shown by the arrows in figure 2 . If this discontinuity, symbolized by $\bar{u}_{1}$, is to continue in an unimpeded manner along the $\beta_{-}$characteristic, any discontinuity in $u_{2}$, symbolized by $\bar{u}_{2}$, must vanish where the two characteristics intersect. Thus, for the propagation of the discontinuity $\bar{u}_{1}$ along $S_{1}$, we have from equation (49a)

$$
\frac{d \bar{u}_{1}}{d \alpha}+\bar{u}_{1} \tan \phi=0
$$

By a similar argument for the $\beta_{+}$characteristic, we find that for the propagation of the discontinuity $\bar{u}_{2}$ along $S_{2}$,

$$
\frac{d \bar{u}_{2}}{d \alpha}+\bar{u}_{2} \tan \phi=0
$$

Integrating equations (50) and (51) yields

$$
\bar{u}_{1}=\bar{u}_{1_{0}} \exp \left[-\left(\alpha-\alpha_{0}\right) \tan \phi\right]
$$

for propagating tangential velocity discontinuities along a $\beta_{-}$characteristic and

$$
\bar{u}_{2}=\bar{u}_{2_{0}} \exp \left[-\left(\alpha-\alpha_{0}\right) \tan \phi\right]
$$

for propagating tangential velocity discontinuities along a $\beta_{+}$characteristic. Here, $\bar{u}_{1_{0}}$ and $\bar{u}_{2_{0}}$ are known tangential velocity discontinuities at some $\alpha=\alpha_{0}$ along $S_{1}$ or $S_{2}$.

In Coulomb materials $(\phi \neq 0)$, discontinuities in velocity can also occur in directions normal to the characteristics $\beta_{-}$and $\beta_{+}$. Defining the normal velocity components $v_{1}$ and $v_{2}$ along $S_{1}$ and $S_{2}$, we have for the relationships between these velocity components and the Cartesian velocity components $u$ and $v$

$$
v_{1}=-u \sin (\alpha-\mu)+v \cos (\alpha-\mu)
$$

and

$$
v_{2}=-u \sin (\alpha+\mu)+v \cos (\alpha+\mu) .
$$

Inverting the pair of equations (46a) and (54a), we arrive at

$$
u=u_{1} \cos (\alpha-\mu)-v_{1} \sin (\alpha-\mu)
$$




$$
v=u_{1} \sin (\alpha-\mu)+v_{1} \cos (\alpha-\mu)
$$

for the $\beta_{-}$characteristic. Inverting equations (46b) and (54b), we find

$$
u=u_{2} \cos (\alpha+\mu)-v_{2} \sin (\alpha+\mu)
$$

and

$$
v=u_{2} \sin (\alpha+\mu)+v_{2} \cos (\alpha+\mu)
$$

for the $\beta_{+}$characteristic.

Substituting equations $(55 \mathrm{a}, \mathrm{b})$ in equation $(45 \mathrm{a})$, and equations $(56 \mathrm{a}, \mathrm{b})$ in equation $(45 \mathrm{~b})$, we find the canonical equations describing the relationships between the tangential and normal velocities along each characteristic:

$$
\frac{\partial u_{1}}{\partial S_{1}}-v_{1} \frac{\partial \alpha}{\partial S_{1}}=0
$$

and

$$
\frac{\partial u_{2}}{\partial S_{2}}-v_{2} \frac{\partial \alpha}{\partial S_{2}}=0
$$

We can also write equations $(57 a, b)$ as the ordinary differential equations

$$
v_{1}=\frac{d u_{1}}{d \alpha}
$$

and

$$
v_{2}=\frac{d u_{2}}{d \alpha}
$$

If a discontinuity in $u_{1}$, represented by $\bar{u}_{1}$, occurs along the $\beta_{-}$characteristic, there will then be a corresponding discontinuity in $v_{1}$, represented by $\bar{v}_{1}$, which is given by substituting equation (50) in equation (58a), or

$$
\bar{v}_{1}=-\bar{u}_{1} \tan \phi .
$$

Similarly, substituting equation $(50)$ in equation $(58 \mathrm{~b})$ yields

$$
\bar{v}_{2}=\bar{u}_{2} \tan \phi .
$$

Then, from equations (52) and (53), we determine that

$$
\bar{v}_{1}=-\tan \phi \bar{u}_{1_{0}} \exp \left[-\left(\alpha-\alpha_{0}\right) \tan \phi\right]
$$

and

$$
\bar{v}_{2}=\tan \phi \bar{u}_{2_{0}}\left[\exp \left(\alpha-\alpha_{0}\right) \tan \phi\right],
$$

which are the relationships for propagating normal velocity discontinuities along the $\beta_{-}$and $\beta_{+}$ characteristics. For a material where $\phi=0$, we see that $\bar{v}_{1}=\bar{v}_{2}=0$ and only tangential-velocity discontinuities $\bar{u}_{1}$ and $\bar{u}_{2}$ are propagated along the $\beta_{-}$and $\beta_{+}$ characteristics. Finally, from equations (59) and (60), we see that the resultant jump in velocity on either characteristic is at an angle $\phi$ to the characteristic at every point.

Equations (52), (53), (59), (60), (61), and (62) are originally due to Shield (1953), and were discussed by Pariseau (1970) and by Houlsby and Wroth (1980). Physically, velocity discontinuities are manifested as zones of intense shear along which volume expansion (dilatancy) and slip occur (Drucker and Prager, 1952). The normal component of velocity discontinuity controls the amount of dilatancy (equations (61) and (62)). In general, velocity discontinuities originate at boundaries between two plastic regions, or between plastic and nonplastic regions-that is, along lines of discontinuity-and propagate into the plastically deforming mass along the velocity characteristics in an exponential manner (equations (52), (53), (61), and (62)).

We are now in a position to proceed to the landslide model, but, before doing so, let us summarize some of the salient points about Coulomb plastic materials. We have developed general differential equations for stress and velocity fields in two-dimensional Coulomb plastic materials and found them to be hyperbolic and integrable by the method of characteristics. Also, we have found that the surfaces along which discontinuities in velocities and deformation rates may occur (velocity characteristics) and the surfaces along which derivatives in stress may be discontinuous (stress characteristics) are coincident. Also, we have seen that, if $\phi \neq 0$, the material must dilate. Finally, because these surfaces are potential paths for propagation of velocity discontinuities, we have termed them slip surfaces and found that velocity discontinuities grow and decay along them exponentially with changes in the angle $\alpha$ between the maximum compressive principal stress and the $x$ axis.

\section{THE LANDSLIDE MODEL}

\section{DETERMINATION OF THE STRESS FIELD}

Our idealized landslide is assumed to form with a definite basal slip plane at depth $y^{*}$, in the semi-infinite, isotropic, and homogeneous mass of soil inclined at an angle $\theta$ to the horizontal as shown in figure 3 . The soil mass, acted upon by gravity, fails according to 


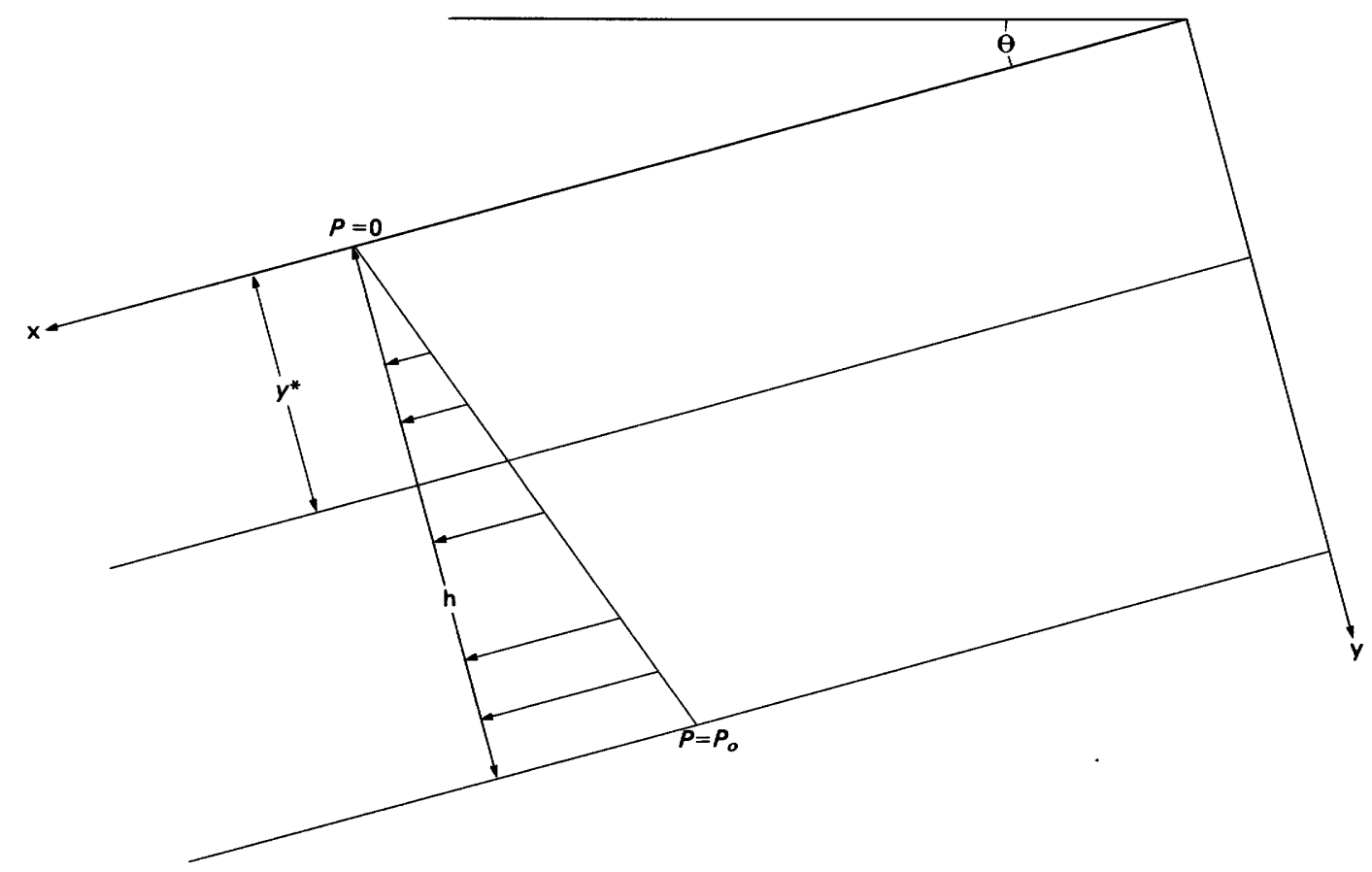

FIGURE 3.-Sketch showing semi-infinite mass of soil inclined at an angle $\theta$ to the horizontal. The linearly varying pore pressure $P$ and the predicted slide depth $y^{*}$ are also shown.

Coulomb's criterion (equation (1)), and a pore pressure $P$ is present, which varies linearly from a depth $y=h$ to the surface $y=0$, or

$$
P=P_{\mathrm{o}} y / h \text {. }
$$

The depth $h$ is considered to be equal to, or greater than, the depth of the basal slip plane of the landslide.

Under these conditions, the stresses can be taken as functions of $y$ only, and the equations of equilibrium (equations $(14 a, b)$ ) can be directly integrated to give

$$
\sigma_{x y}=\rho g y \sin \theta
$$

and

$$
\sigma_{y}^{\prime}=\rho g y(\cos \theta-\bar{P}),
$$

where $\bar{P}=P_{\mathrm{o}} / \rho g h$ is the dimensionless pore pressure.

For the soil in the inclined half-space to be in a state of limiting equilibrium, we have from equations $(9 a, b)$

$$
\sigma_{x y}=\rho g y \sin \theta=\sigma^{\prime} \sin \phi \sin 2 \alpha
$$

and

$\sigma_{y}^{\prime}=\rho g y(\cos \theta-\bar{P})=\sigma^{\prime}(1-\sin \phi \cos 2 \alpha)-H,(65 b)$

from which we can solve for $\sigma^{\prime}$ and $y$ by inverting $\left[\begin{array}{cc}1-\sin \phi \cos 2 \alpha & -\rho g(\cos \theta-\bar{P}) \\ \sin \phi \sin 2 \alpha & -\rho g \sin \theta\end{array}\right]\left[\begin{array}{l}\sigma^{\prime} \\ y\end{array}\right]=\left[\begin{array}{c}H \\ 0\end{array}\right]$.

Carrying out the inversion, we have

$$
\sigma^{\prime}=\frac{H \sin \theta}{\sin \theta-\sin \phi \sin (2 \alpha+\theta)+\bar{P} \sin \phi \sin 2 \alpha}
$$

and

$$
y=\frac{H \sin \phi \sin 2 \alpha}{\rho g[\sin \theta-\sin \phi \sin (2 \alpha+\theta)+\bar{P} \sin \phi \sin 2 \alpha]} .
$$

For the sake of brevity in future equations, we shall define the recurring terms $\eta$ and $D_{1}$ :

and

$$
\eta=(2 \alpha+\theta)
$$

$D_{1}=\sin \theta-\sin \phi \sin \eta+\bar{P} \sin \phi \sin 2 \alpha$.

The validity of the solutions (67) and (68) is limited. For equilibrium of the soil medium, we see from figure 1 that

$$
\tau_{n} \leqslant\left(\sigma_{n}^{\prime}+H\right) \tan \phi
$$

or, in this case, where $\tau_{n}=\sigma_{x y}=\rho g y \sin \theta$, and $\sigma_{n}^{\prime}=\sigma_{y}^{\prime}=\rho g y(\cos \theta-\bar{P})$, that 
$\rho g y[\sin \theta-(\cos \theta-\bar{P}) \tan \phi] \leqslant H \tan \phi$.

(72)

Since $k=H \tan \phi$ by equation (2), relation (72) can be written

$$
y \leqslant \frac{k \cos \phi}{\rho g[\sin (\theta-\phi)+\bar{P} \sin \phi]} .
$$

The upper limit

$$
y=y^{*}=\frac{k \cos \phi}{\rho g[\sin (\theta-\phi)+\bar{P} \sin \phi]},
$$

where $\sigma_{x y}=k+\sigma_{y}^{\prime} \tan \phi$-that is, where Coulomb's criterion is satisfied-represents the limit to the validity of solutions (67) and (68).

Thus, when $\sin (\theta-\phi)+\bar{P} \sin \phi>0$, limiting equilibrium is possible only in the strip $0 \leqslant y \leqslant y^{*}$. If $\sin (\theta-\phi)+\bar{P} \sin \phi=0$, then $y^{*} \rightarrow \infty$. Stress fields and characteristics for $y^{*} \rightarrow \infty$ were discussed by Terzaghi (1943) and Sokolovskii $(1960,1965)$. Equation (74) clearly shows that $\mathrm{y}^{*}$ will occur at a finite depth when $\theta=\phi$ and $\bar{P}$ is positive. When $\sin (\theta-\phi)+\bar{P} \sin \phi$ $<0$ a stable slope occurs, as the slide plane is predicted to be above the slope surface.

In figure 4 , the depth $y^{*}$ from equation (74) is plotted in dimensionless form $\rho g y^{*} / k$ as a function of the

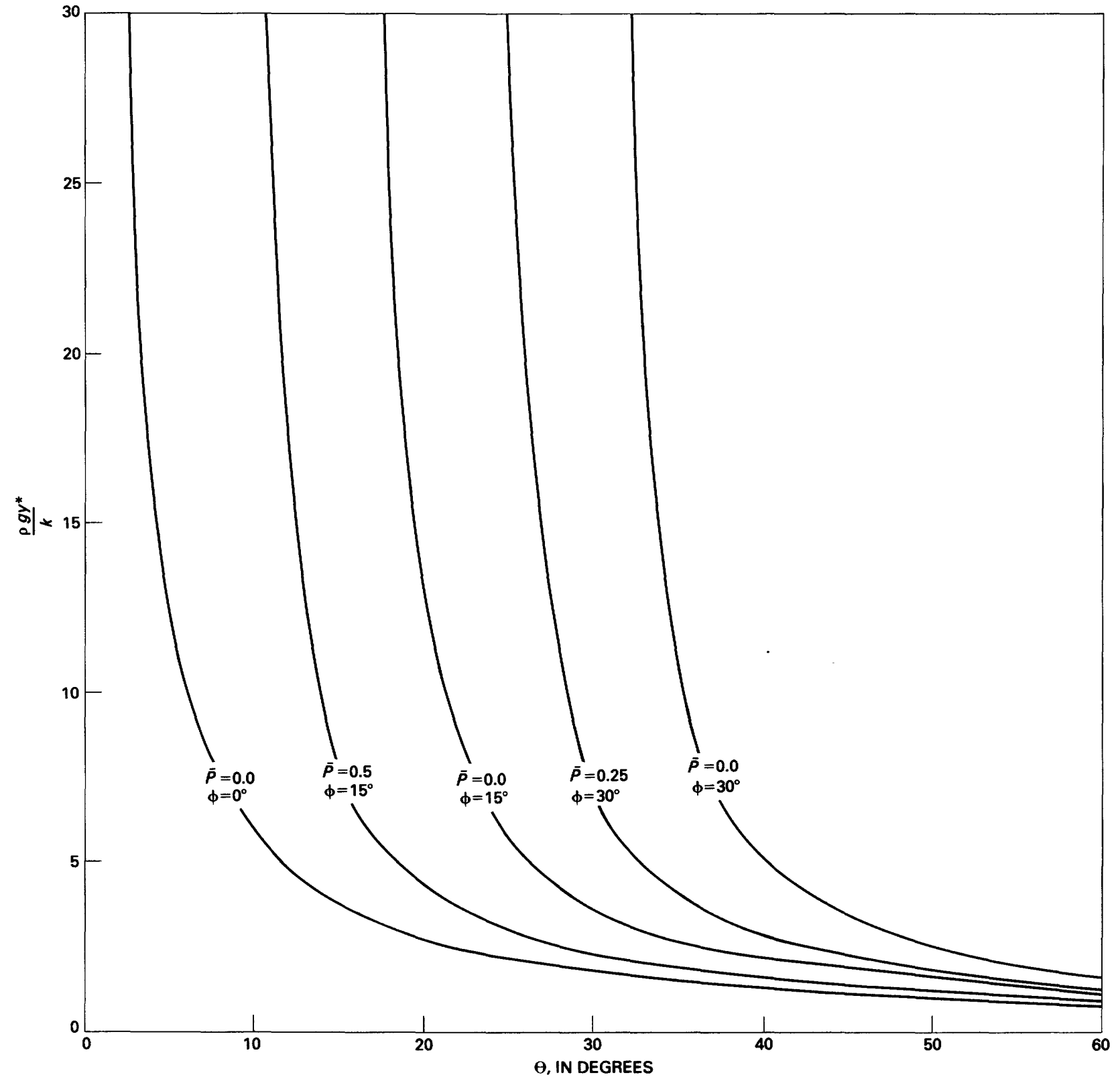

FIGURE 4.-Graph showing $\frac{\rho g y^{*}}{k}$ versus $\theta$ for various values of $\bar{P}$ and $\phi$. 
slope angle $\theta$ for various values of $\bar{P}$ and $\phi$. From equation (74), we see that $y^{*}$ increases with increasing cohesion $k$ and decreases with increasing density $\rho$. The gravitational acceleration $g$ is assumed constant. From figure 4, we see that variations in $\bar{P}$ have no effect on $y^{*}$ when $\phi=0$. In this purely cohesive case, $y^{*}$ depends only on $\theta, \rho$, and $k$. When $\phi$ and $\theta$ are constant $(\phi>0)$, the depth of the landslide $y^{*}$ decreases as the dimensionless pore pressure $\bar{P}$ increases. This dependence on pore pressure is expected from Coulomb's criterion and the effective stress law, as a reduction in normal stress $\sigma_{n}$ requires a corresponding reduction in the maximum shear stress $\sigma_{x y}=\rho g y^{*} \sin \theta$, and hence a reduction of $y^{*}$. Also, for constant $\bar{P}$ and $\theta, y^{*}$ increases as $\phi$ is increased. Finally, as $\theta$ increases, the curves in figure 4 converge, the dependence of $y^{*}$ on $\phi$ and $\bar{P}$ lessens, and the depth of the landslide approaches that for pure cohesion.

For limiting equilibrium to be possible in the strip $0 \leqslant y \leqslant y^{*}$, the dimensionless pore pressure $\bar{P}$ in equation
(74) must be restricted. Let us rewrite equation (74) as

$$
y^{*}=\frac{k \cot \phi}{\rho g[\sin \theta \cot \phi-\cos \theta+\bar{P}]} .
$$

If $\bar{P}=\cos \theta-\sin \theta \cot \phi$, then $y^{*} \rightarrow \infty$. If $\bar{P}=\cos \theta$, then from equation $(64 \mathrm{~b}) \sigma_{y}^{\prime}=\rho g y(\cos \theta-\bar{P})=0$, and there will be no normal stress on any plane parallel to $y^{*}$ in $0 \leqslant y \leqslant y^{*}$. If $\bar{P}>\cos \theta$, there will be tensile normal stresses on all planes parallel to $y^{*}$, a case not covered by the Coulomb criterion. Thus, the limits on $\bar{P}$ for $\phi>0$ are given by

$$
\cos \theta-\sin \theta \cot \phi \leqslant \bar{P} \leqslant \cos \theta .
$$

These bounds on $\bar{P}$ as functions of $\theta$ and $\phi$ are shown in figure 5. For $\phi=0, \bar{P}$ is taken to have the range $0 \leqslant \bar{P} \leqslant \cos \theta$.

If we rewrite equation (74) as

$$
\frac{k \cos \phi}{\rho g}=y^{*}[\sin (\theta-\phi)+\bar{P} \sin \phi] \text {. }
$$

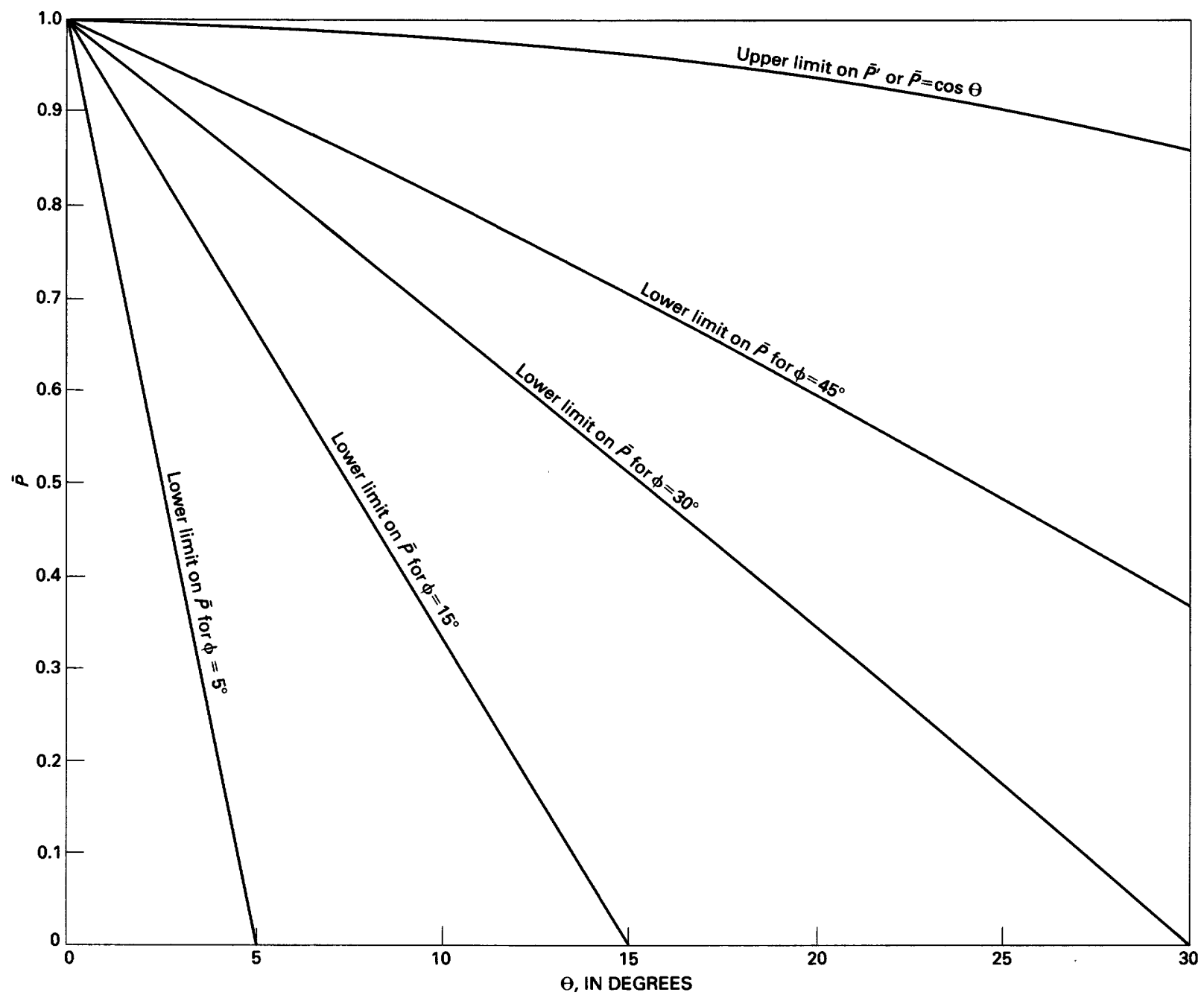

FIGURE 5.-Graph showing bounds on $\bar{P}$ as a function of slope angle $\theta$ for various angles of internal friction $\phi$. 
equation (68) becomes

$$
y=\frac{y^{*}[\sin (\theta-\phi)+\bar{P} \sin \phi] \sin 2 \alpha}{D_{1}} .
$$

If $\alpha=45^{\circ}-\frac{\phi}{2}$ is substituted in (78), it reduces to $y=y^{*}$; and, hence the line $y=y^{*}$ represents a line of discontinuity along which the slopes $\left[\frac{d y}{d x}\right]_{ \pm}$given by equation (20) are respectively infinite and zero. Because stresses undergo finite jumps and stress gradients are infinite as we cross a line of discontinuity, the equations describing limiting equilibrium in the soil mass are valid only when $0 \leqslant y \leqslant y^{*}$. The line $y=y^{*}$ represents the basal slip plane of the landslide.

We have equations $(65 \mathrm{a}, \mathrm{b})$ for $\sigma_{x y}$ and $\sigma_{y}^{\prime}$ in our idealized landslide. We can find the expression for $\sigma_{x}^{\prime}$ by substituting equation (67) into equation (9a), which yields

$$
\sigma_{x}^{\prime}=\frac{H \sin \theta(1+\sin \phi \cos 2 \alpha)}{D_{1}}-H .
$$

For future convenience, we shall write equations $(65 a, b)$ and $(79)$ in the dimensionless forms

$$
\begin{gathered}
\bar{\sigma}_{x y}=\frac{\sigma_{x y}}{k}=\frac{\rho g y}{k} \sin \theta=\frac{\sin \theta \cos \phi \sin 2 \alpha}{D_{1}}, \\
\bar{\sigma}_{y}^{\prime}=\frac{\sigma_{y}^{\prime}}{k}=\frac{\rho g y}{k}(\cos \theta-\bar{P})=\frac{\cos \phi(\cos \theta-\bar{P}) \sin 2 \alpha}{D_{1}},
\end{gathered}
$$

and

$$
\bar{\sigma}_{x}^{\prime}=\frac{\sigma_{x}^{\prime}}{k}=\frac{\cos \phi[(\cos \theta-\bar{P}) \sin 2 \alpha+2 \cos 2 \alpha \sin \theta]}{D_{1}},
$$

which are valid for $0 \leqslant y^{\prime} \leqslant 1$, where $y^{\prime}=y / y^{*}$-that is, between the ground surface and the basal slip plane of the landslide.

When $\phi=0$, equations (80), (81), and (82) reduce to

$$
\begin{gathered}
\bar{\sigma}_{x y}=\frac{\sigma_{x y}}{k}=\frac{\rho g y \sin \theta}{k}=\sin 2 \alpha, \\
\bar{\sigma}_{y}^{\prime}=\frac{\sigma_{y}^{\prime}}{k}=\frac{\rho g y(\cos \theta-\bar{P})}{k}=\frac{[\cos \theta-\bar{P}]}{\sin \theta} \sin 2 \alpha,
\end{gathered}
$$

and

$$
\bar{\sigma}_{x}^{\prime}=\frac{\sigma_{x}^{\prime}}{k}=\frac{(\cos \theta-\bar{P}) \sin 2 \alpha}{\sin \theta}+2 \cos 2 \alpha ;
$$

and equation (78) reduces to

$$
y=y^{*} \sin 2 \alpha
$$

or

$$
\sin 2 \alpha=y / y^{*}
$$

from which

$$
\cos 2 \alpha= \pm\left[1-\left(y / y^{*}\right)^{2}\right]^{1 / 2}
$$

The stress field for the purely cohesive case can then be given in the alternate dimensionless forms

$$
\begin{gathered}
\bar{\sigma}_{x y}=y / y^{*}, \\
\bar{\sigma}_{y}^{\prime}=\frac{(\cos \theta-\bar{P})}{\sin \theta}\left(y / y^{*}\right),
\end{gathered}
$$

and

$$
\bar{\sigma}_{x}^{\prime}=\frac{(\cos \theta-\bar{P})}{\sin \theta}\left(y / y^{*}\right) \pm 2\left[1-\left(y / y^{*}\right)^{2}\right]^{1 / 2},
$$

where the upper sign in the last equation is taken for compressive flow. These equations for $\bar{\sigma}_{x}^{\prime}, \bar{\sigma}_{y}^{\prime}$, and $\bar{\sigma}_{x y}$ are, with notational and coordinate axis differences, similar to those presented by Nye (1951, equations 8 , p. 558) for stresses in a purely cohesive alpine glacier. There are two sets of limits on $\alpha$, the angle that the greatest principal effective stress $\sigma_{1}^{\prime}$ makes with the $x$ axis. The first set is for compressive (passive) flow when $\alpha=0$ is at the surface and $\sigma_{1}^{\prime}$ is oriented parallel to the $x$ axis at $y=0$. The limits on $\alpha$ for $0 \leqslant y^{\prime} \leqslant 1$ are then

$$
0 \leqslant \alpha \leqslant 45^{\circ}-\phi / 2 \text {, }
$$

where the upper limit is the value of $\alpha$ on the line of discontinuity at $y=y^{*}$. The second set is for extending (active) flow when $\alpha$ at the surface is $90^{\circ}$ and $\sigma_{1}^{\prime}$ is normal to the ground surface at $y=0$. The limits on $\alpha$ for extending flow, $0 \leqslant y^{\prime} \leqslant 1$ are then

$$
45^{\circ}-\frac{\phi}{2} \leqslant \alpha \leqslant 90^{\circ},
$$

where the lower limit is the value of $\alpha$ at $y=y^{*}$. Note 
that these limits apply in the purely cohesive case when $\phi$ is set to zero in equations (86) and (87).

Compressive (passive) flow would be expected where the ground surface is concave upward (where the slope is decreasing). Extending (active) flow would be expected where the ground surface is convex upward (as over a local topographic high on the slope).

Figure 6 shows the variation of $\bar{\sigma}_{x}^{\prime}, \bar{\sigma}_{y}^{\prime}$, and $\bar{\sigma}_{x y}$ with $y / y^{*}$ in compressive and extending flow for $\theta \stackrel{x y}{=} 20^{\circ}$, $\phi=30^{\circ}$, and $\bar{P}=0.5$. For various values of $\phi, \theta$, and $\bar{P}$, the stress distribution will have this general appearance so it will suffice to investigate some of its predominant features.

As we can see from the first forms of equations (80) and (81), the shear stress $\bar{\sigma}_{x y}$ and the vertical normal effective stress $\bar{\sigma}_{y}^{\prime}$ increase linearly with depth to a maximum at the basal slip plane of the landslide $(y=$ $\left.y^{*}\right)$. Figures 7, 8, and 9 show the variation of $\bar{\sigma}_{x y}, \bar{\sigma}_{y}^{\prime}$, and $\bar{\sigma}_{x}^{\prime}$ on $y=y^{*}$ with $\theta$ for various values of $\phi$ and $\bar{P}$. These curves, obtained from the second forms of equations $(80)$ and $(81)$ and equation $(82)$, show that the shear and normal stresses at the landslide's basal slip plane decrease as the pore pressure $\bar{P}$ increases for a given $\theta$ and $\phi$. This decrease is expected from the effective stress law. Also, we see that increasing $\phi$ increases each stress component for a given value of $\bar{P}$ and $\theta$. This increase happens because the stresses depend on the depth of the landslide, which increases as $\phi$ is increased (fig. 4). Also, for the same reason, the stresses on $y=y^{*}$ increase without limit as $\theta$ approaches the value that makes $y^{*}$ infinite (equation (74)) for particular values of $\phi$ and $\bar{P}$. Finally, as the slope angle increases, the stresses on the basal slip plane decrease, become less dependent on $\phi$ and $\bar{P}$, and approach those in the purely cohesive case. The stresses on the basal slip plane decrease because the landslide gets thinner as the slope angle increases (fig. 4). As the landslide gets thinner, the mean stress on $y=y^{*}$ is reduced, and by the Coulomb criterion (fig. 1) the maximum allowable shear stress must be reduced. However, note that in the purely cohesive case, the shear stress $\sigma_{x y}$ on $y=y^{*}$ is always equal to the cohesion $k$.

When $y=0$, equation (82) reduces to

$$
\bar{\sigma}_{x}^{\prime}=\frac{2 \cos \phi}{1-\sin \phi}
$$

for compressive flow ( $\alpha=0$ when $y=0$ ), and to

$$
\bar{\sigma}_{x}^{\prime}=\frac{-2 \cos \phi}{1+\sin \phi}
$$

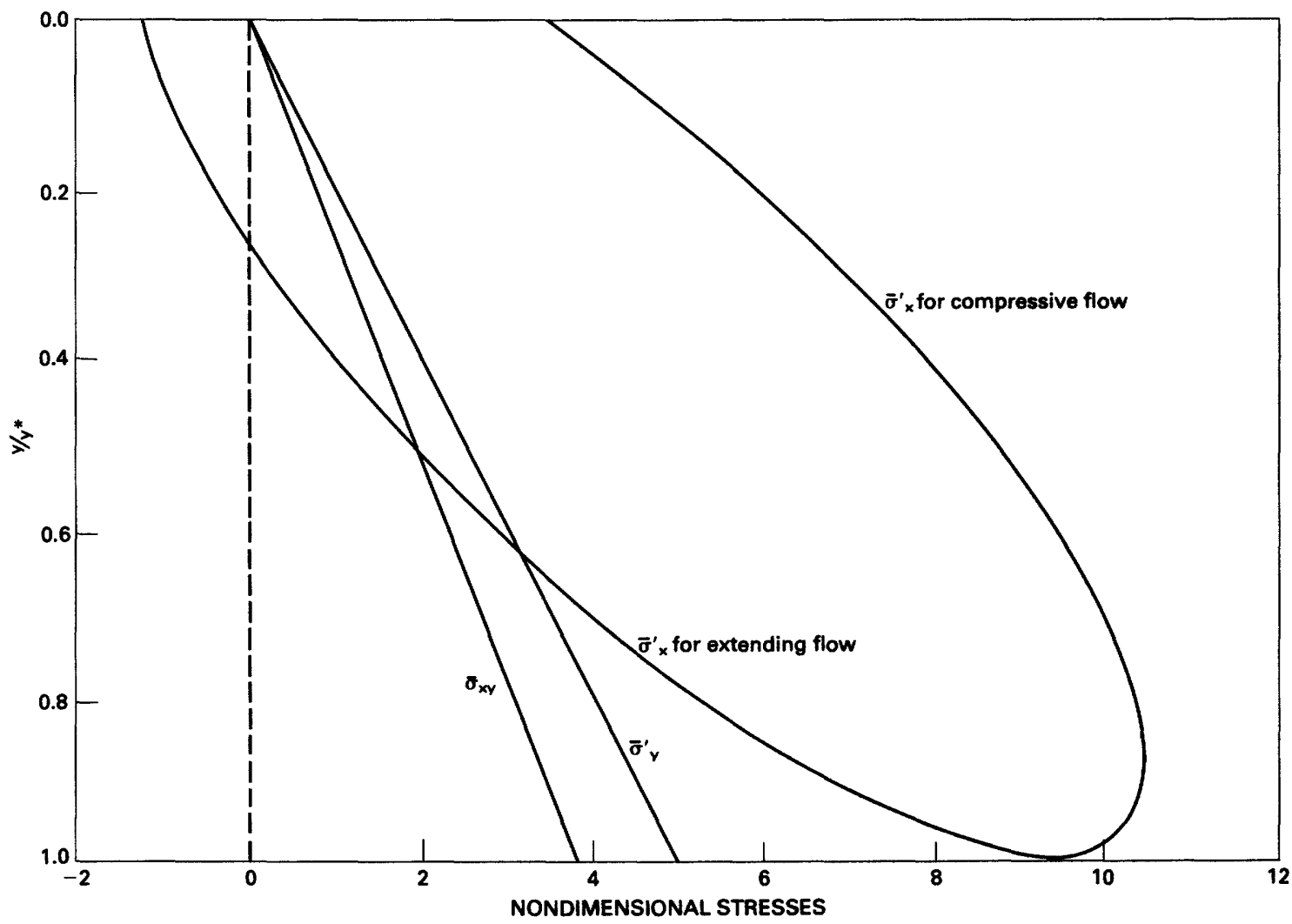

FIGURE 6.-Graph showing variation of $\bar{\sigma}_{x}^{\prime}, \bar{\sigma}_{x y}$, and $\bar{\sigma}_{y}^{\prime}$ with $y / y^{*}$ for $\phi=30^{\circ}, \theta=20^{\circ}$, and $\bar{P}=0.5$ in extending and compressive flow. 


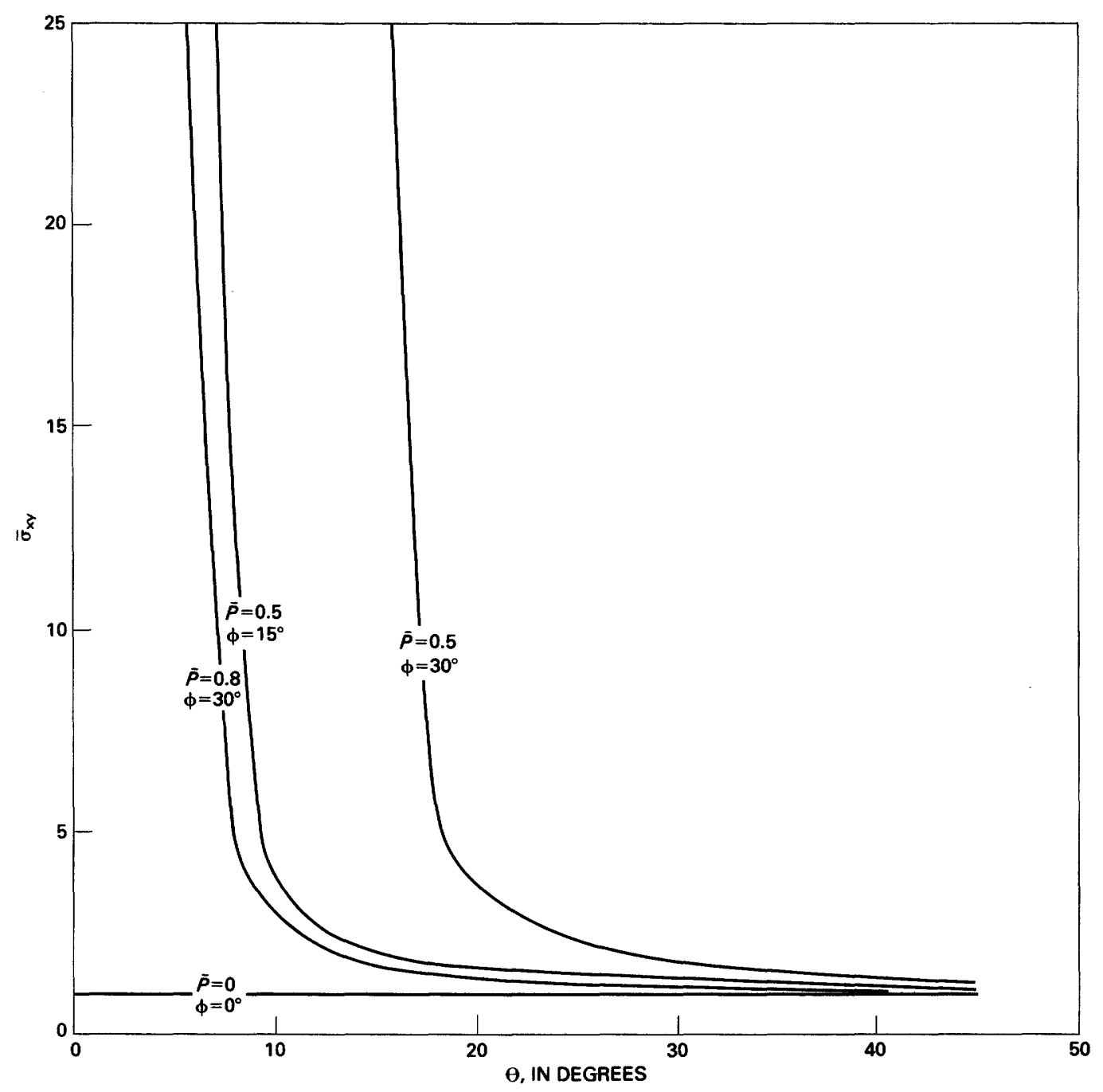

FIGURE 7.-Graph showing variation of $\bar{\sigma}_{x y}$ at $y=y^{*}$ with $\theta$ for various values of $\bar{P}$ and $\phi$.

for extending flow $\left(\alpha=90^{\circ}\right.$ when $\left.y=0\right)$. Thus, when $y=0, \bar{\sigma}_{x}^{\prime}$ depends only on $\phi$ and becomes more compressive (less tensile) as $\phi$ increases, as shown in figure 10.

Figure 6 shows that $\bar{\sigma}_{x}^{\prime}$ is tensile to a certain depth in extending flow. By solving equation (82) for $\alpha$ when $\bar{\sigma}_{x}^{\prime}=0$, we find that $\alpha$ is given by

$$
\alpha=45^{\circ}+\frac{1}{2} \tan ^{-1}\left(\frac{\cos \theta-\bar{P}}{2 \sin \theta}\right),
$$

where $\alpha$ lies within the limits given by relation (87). The value of $\alpha$ computed from equation (90) is then substituted in equation (78) to give the depth $y / y^{*}$ to which $\bar{\sigma}_{x}^{\prime}$ is tensile in extending flow. Figure 11 shows this depth as a function of $\theta$ for various values of $\bar{P}$ and $\phi$. From figure 11 , we see that the depth to which $\bar{\sigma}_{x}^{\prime}$ is tensile in extending flow increases with slope angle and pore pressure but decreases as internal friction increases.
Figure 6 shows that $\bar{\sigma}_{x}^{\prime}$ is a maximum at a certain depth in compressive flow. By differentiating equation (82), setting the result equal to zero, and solving for $\alpha$ we find that

$$
\begin{gathered}
\alpha=\tan ^{-1}\left[\frac{2 \sin \theta}{(\bar{P}-\cos \theta)(1-\sin \phi)}\right. \\
\left.-\frac{\left[4 \sin ^{2} \theta+\cos ^{2} \phi(\bar{P}-\cos \theta)^{2}\right]^{1 / 2}}{(\bar{P} \cdot \cos \theta)(1-\sin \phi)}\right],
\end{gathered}
$$

subject to relation (86), for $\bar{\sigma}_{x}^{\prime}$ to be a maximum in compressive flow. This value of $\alpha$ is then substituted in equation (78) to give the depth where $\bar{\sigma}_{x}^{\prime}$ is a maximum and substituted in equation (82) to give the magnitude of $\bar{\sigma}_{x}^{\prime}$ at this same depth. Figure 12 shows the depth of the maximum values of $\bar{\sigma}_{x}^{\prime}$ in compressive flow as a 


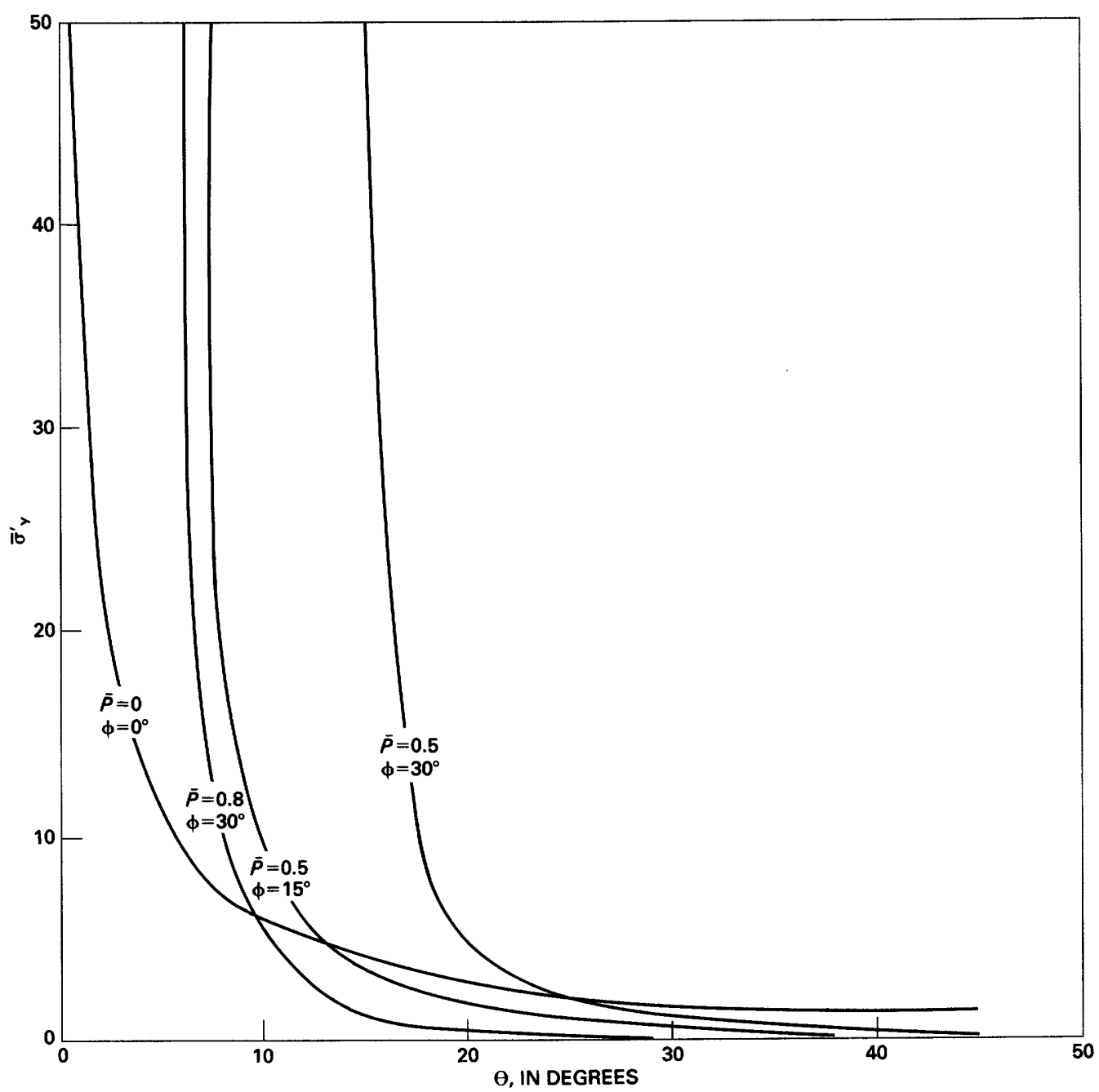

FIGURE 8.-Graph showing variation of $\bar{\sigma}_{y}^{\prime}$ at $y=y^{*}$ with $\theta$ for various values of $\bar{P}$ and $\phi$.

function of $\theta$ for various values of $\phi$ and $\bar{P}$. Note that this depth decreases with increasing slope angle and pore pressure, and increases with increasing internal friction. Figure 13 shows the maximum value of $\bar{\sigma}_{x}^{\prime}$ in compressive flow as a function of $\theta$ for various values of $\bar{P}$ and $\phi$. Like the other stress values shown in figures 7,8 , and 9 , the maximum values of $\bar{\sigma}_{x}^{\prime}$ in compressive flow decrease with increasing pore pressure and slope angle, and become larger with increasing internal friction. Note that here, $\bar{\sigma}_{x}^{\prime}$ also becomes infinite as $\theta$ approaches the value that makes $y^{*}$ infinite and the dependence of the maximum $\bar{\sigma}_{x}^{\prime}$ on $\phi$ and $\bar{P}$ decreases as $\theta$ increases.

In general then, all dimensionless stresses other than $\bar{\sigma}_{x}^{\prime}$ at the surface (which depends only on $\phi$, as shown in fig. 10) decrease with increasing pore pressure, increasing slope angle, and decreasing internal friction. Of course, the actual stresses (equations (80), (81), and (82)) also increase in direct proportion to the cohesion $k$.
Stress characteristics are determined by integrating equation (20), which can be written in the canonical form

$$
\frac{\partial y}{\partial \alpha}=\tan \left[\alpha \pm\left(45^{\circ}-\frac{\phi}{2}\right)\right] \frac{\partial x}{\partial \alpha} .
$$

Taking the derivative of equation (78) with respect to $\alpha$, we find

$\frac{\partial y}{\partial \alpha}=\frac{2 y^{*}[\sin (\theta-\phi)+\bar{P} \sin \phi] \sin \theta(\cos 2 \alpha-\sin \phi)}{D_{1}^{2}}$

We now define the angle

$$
\delta=(\theta-\phi) .
$$

From equation (92), we find 


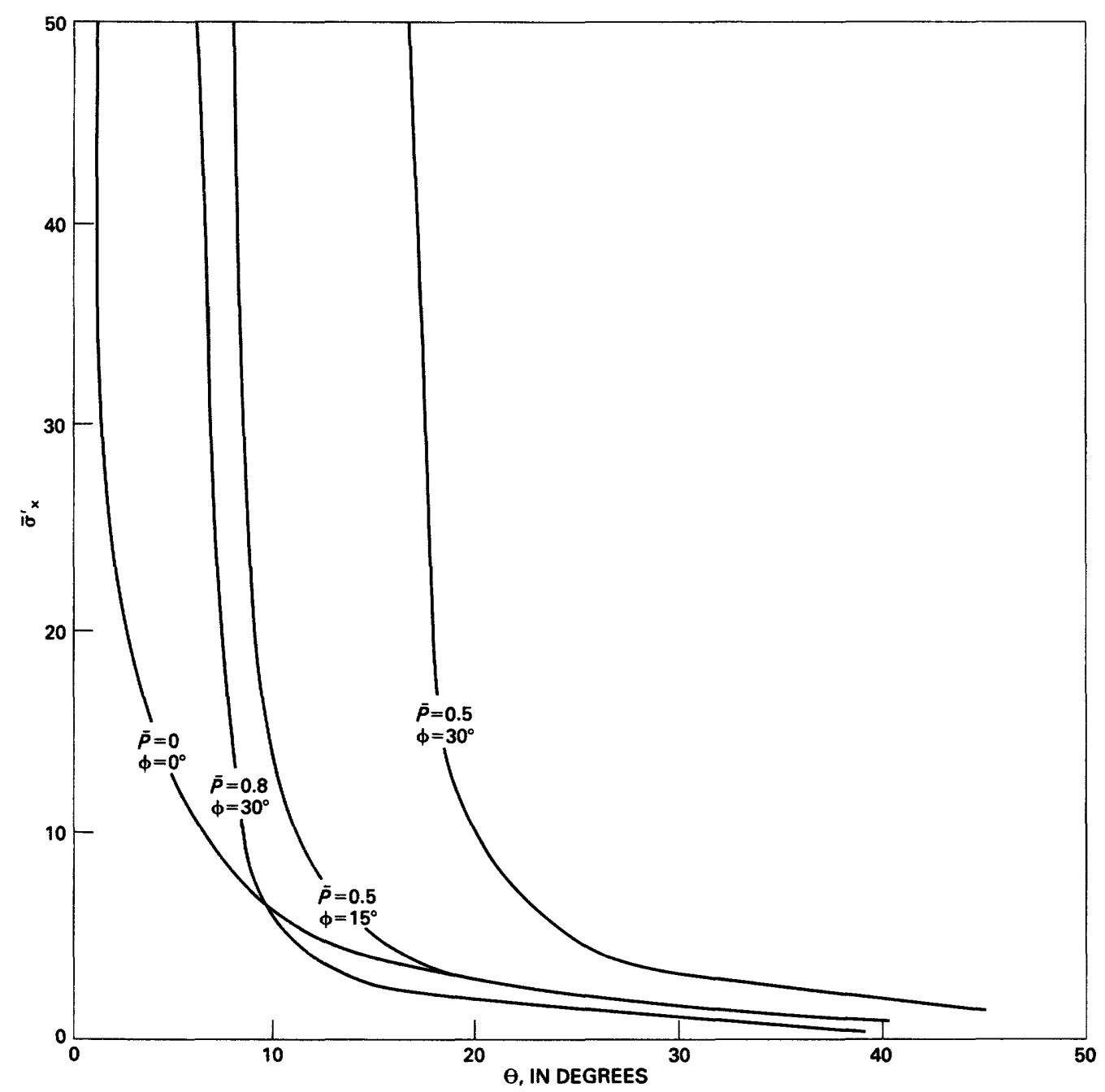

FIGURE 9.-Graph showing variation of $\bar{\sigma}_{x}^{\prime}$ at $y=y^{*}$ with $\theta$ for various values of $\bar{P}$ and $\phi$.

or

$$
\frac{\partial x}{\partial \alpha}=\cot \left[\alpha \pm\left(45^{\circ}-\frac{\phi}{2}\right)\right] \frac{\partial y}{\partial \alpha}
$$

$\frac{\partial x}{\partial \alpha}=\frac{-2 y^{*}[\sin \delta+\bar{P} \sin \phi] \sin \theta(\sin 2 \alpha \mp \cos \phi)}{D_{1}^{2}}$

which is integrated (Gradshteyn and Ryzhik, 1980, p. 149) to give

$$
\begin{gathered}
x=x_{\mathrm{o}}+2 y^{*}[\sin \delta+\bar{P} \sin \phi] \sin \theta \\
\{F[\phi, \theta, \bar{P}]+G[\phi, \theta, \bar{P}]\},
\end{gathered}
$$

where

$-\sin \phi \sin \theta$

$+[\sin \theta \pm \cos \phi \sin \phi(\bar{P}-\cos \theta)] \cos 2 \alpha$

$F[\phi, \theta, \bar{P}]=\frac{ \pm \cos \phi \sin \phi \sin \theta \sin 2 \alpha}{2 D_{2}[\sin \theta-\sin \phi \sin \theta \cos 2 \alpha}$
$G[\phi, \theta, \bar{P}]=\frac{[\sin \phi(\bar{P}-\cos \theta) \pm \cos \phi \sin \theta]}{\left(D_{2}\right)^{3 / 2}}$

$\tan ^{-1}\left|\frac{(\sin \theta+\sin \phi \sin \theta) \tan \alpha+\sin \phi(\bar{P}-\cos \theta)}{\left(D_{2}\right)^{1 / 2}}\right|$

and

$D_{2}=\sin ^{2} \theta-\sin ^{2} \phi \sin ^{2} \theta-\sin ^{2} \phi(\bar{P}-\cos \theta)^{2}$.

(100)

Equation (97) represents the $x$ coordinates of the first (upper signs in the functions $F$ and $G$ ) and second (lower signs in $F$ and $G$ ) characteristic directions and is valid only when

$$
\sin ^{2} \theta>\sin ^{2} \phi \sin ^{2} \theta+\sin ^{2} \phi(\bar{P}-\cos \theta)^{2} .
$$

Relation (101) can be written

$$
(\bar{P}-\cos \theta)^{2}>\sin ^{2} \theta \cot ^{2} \phi,
$$




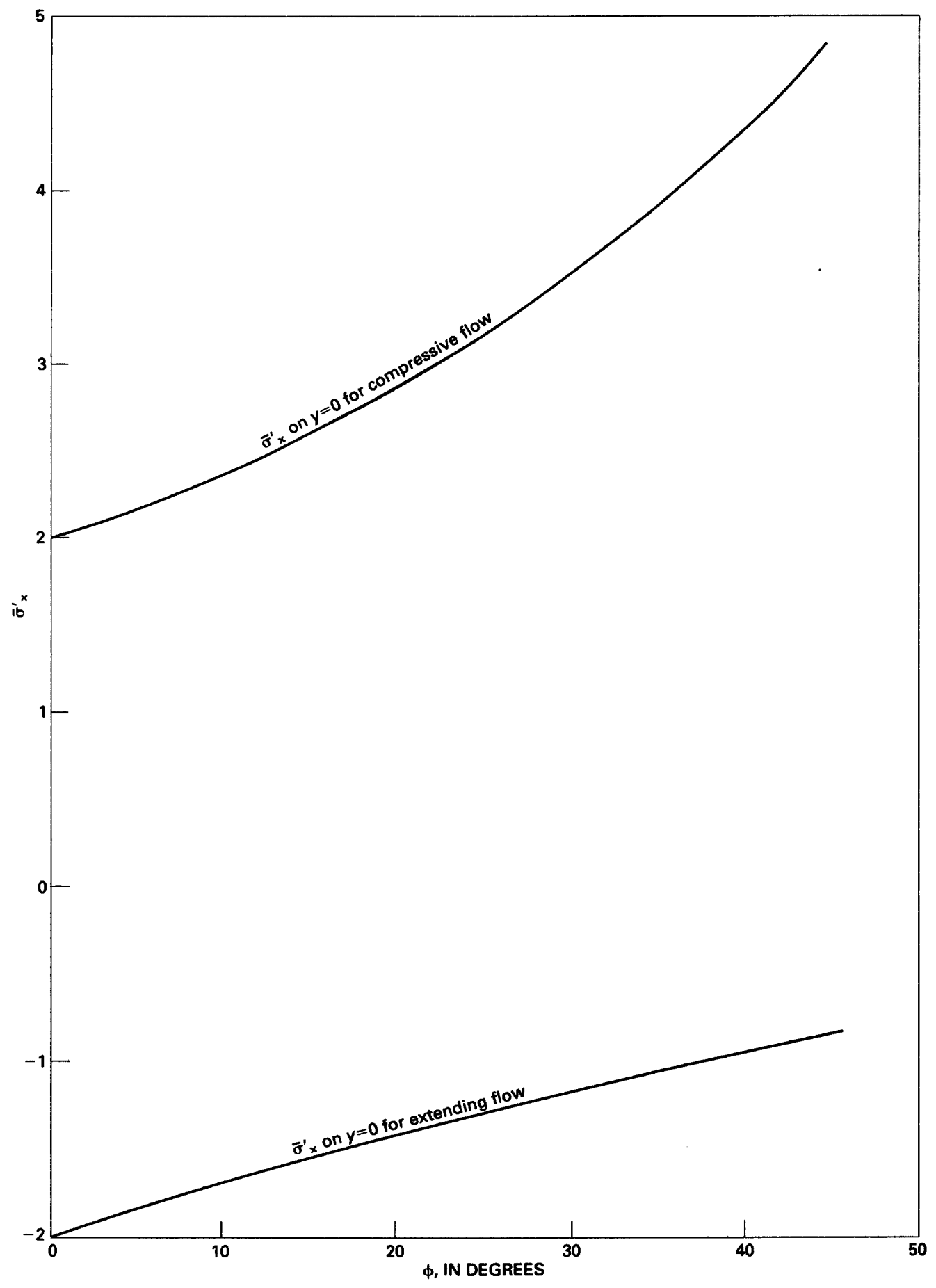

FIGURE 10.-Graph showing variation of $\bar{\sigma}_{x}^{\prime}$ at the surface, $y=0$, with $\phi$.

or

$$
\begin{aligned}
\cos \theta-\sin \theta \cot \phi<\bar{P}< & \cos \theta \\
& +\sin \theta \cot \phi,
\end{aligned}
$$

which is similar to relation (76) if the upper limit on $\bar{P}$ is restricted to $\cos \theta$ (equation (64b)). This restriction $\mid$ and was made on the grounds that the normal stress $\sigma_{y}$ was to be compressive only.

When $\phi=0$, equations (78) and (97) for the stress characteristics reduce to

$$
y=y^{*} \sin 2 \alpha
$$




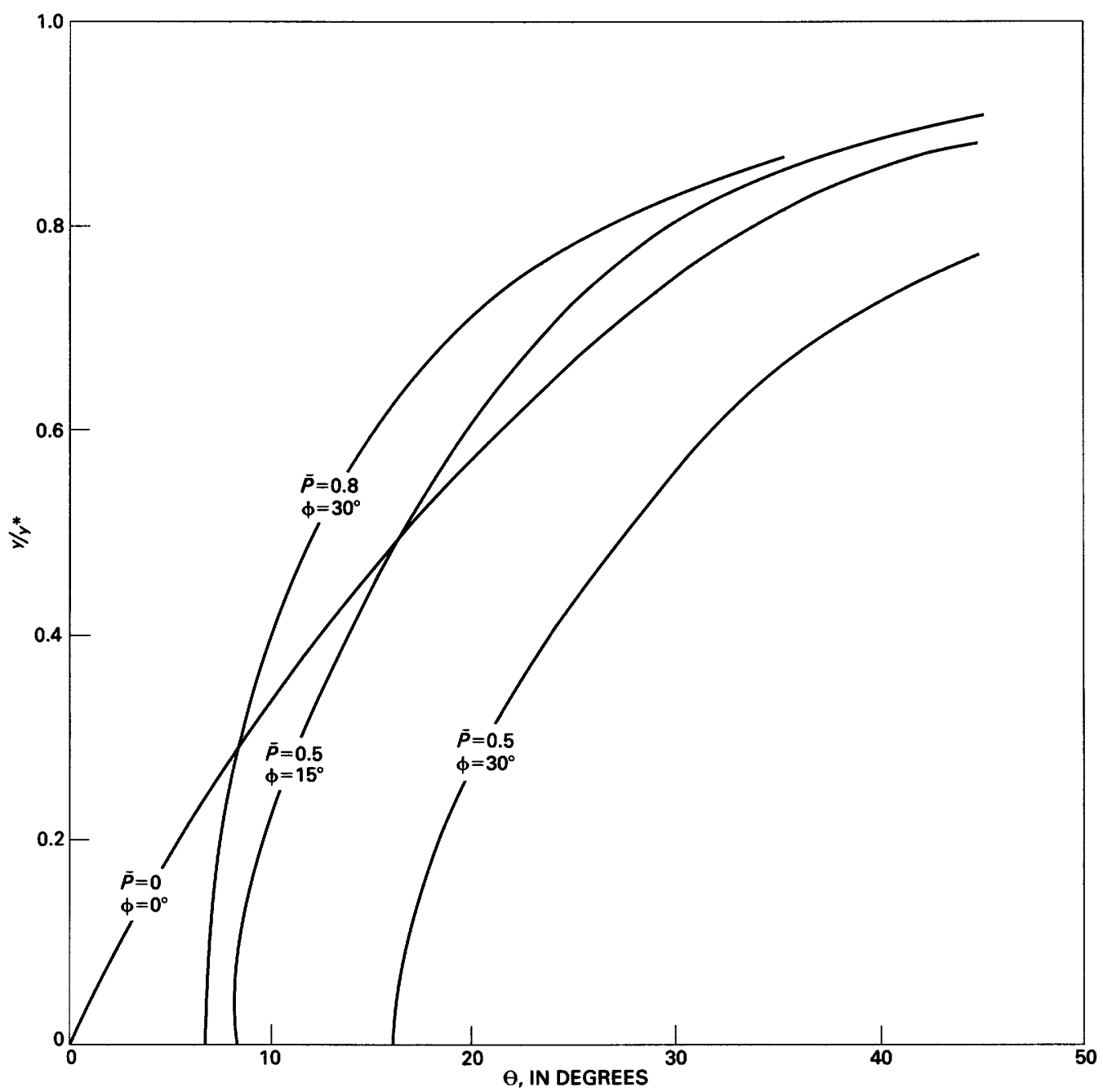

FigURE 11.-Graph showing dimensionless depth $y / y^{*}$ to which $\bar{\sigma}_{x}^{\prime}$ is tensile in extending flow as a function of $\theta$ for various values of $\bar{P}$ and $\phi$.

$$
x=x_{\mathrm{o}}+y^{*} \cos 2 \alpha \pm 2 y^{*} \alpha .
$$

Relation (101) shows that equations (104) and (105) are valid when the slope angle $\theta$ is greater than zero.

Equations (78) and (97) give the $y$ and $x$ coordinates of the stress characteristics. Figure 14 gives a few examples of stress characteristics for various values of $\theta$, $\phi$, and $\bar{P}$ in compressive and extending flow. Comparing figures $14 A$ and $14 E$, we see that increasing $\phi$ for a constant $\theta$ and $\bar{P}$ causes the characteristics to become less curved. We see the same effect as the slope angle $\theta$ is decreased (compare figs. $14 D$ and $14 E$ or figs. $14 A$ and $14 C$. The stress characteristics are also flattened as $\bar{P}$ is decreased (compare figs. $14 B$ and $14 C$ ). The flattening of the stress characteristics is consistent with our observation (fig. 4) that increasing $\phi$ or decreasing $\theta$ or $\bar{P}$ increases the depth $y^{*}$ of the model landslide. In fact, as $y^{*}$ increases without bound, the stress characteristics approach straight lines at depth (Terzaghi, 1943, p. 38). Finally, only some of the stress characteristics for each value of $\theta, \phi$, and $\bar{P}$ are shown in figure 14; but, because of the arbitrary constant of integration $x_{\mathrm{o}}$ in equation (97), there are actually an infinite number of stress characteristics within the deforming regions shown.

As we mentioned before, the stress characteristics in figure 14 intersect at every point at fixed angles dependent on $\phi$. The characteristics are loci along which the shear stress is the limiting shear stress given by equation (1), and loci along which potential discontinuities in stress and stress gradient will be propagated. Also, the basal slip planes in figure $14 A$ through $14 F$ form envelopes to the second set of stress characteristics and, hence, are lines of discontinuity. 


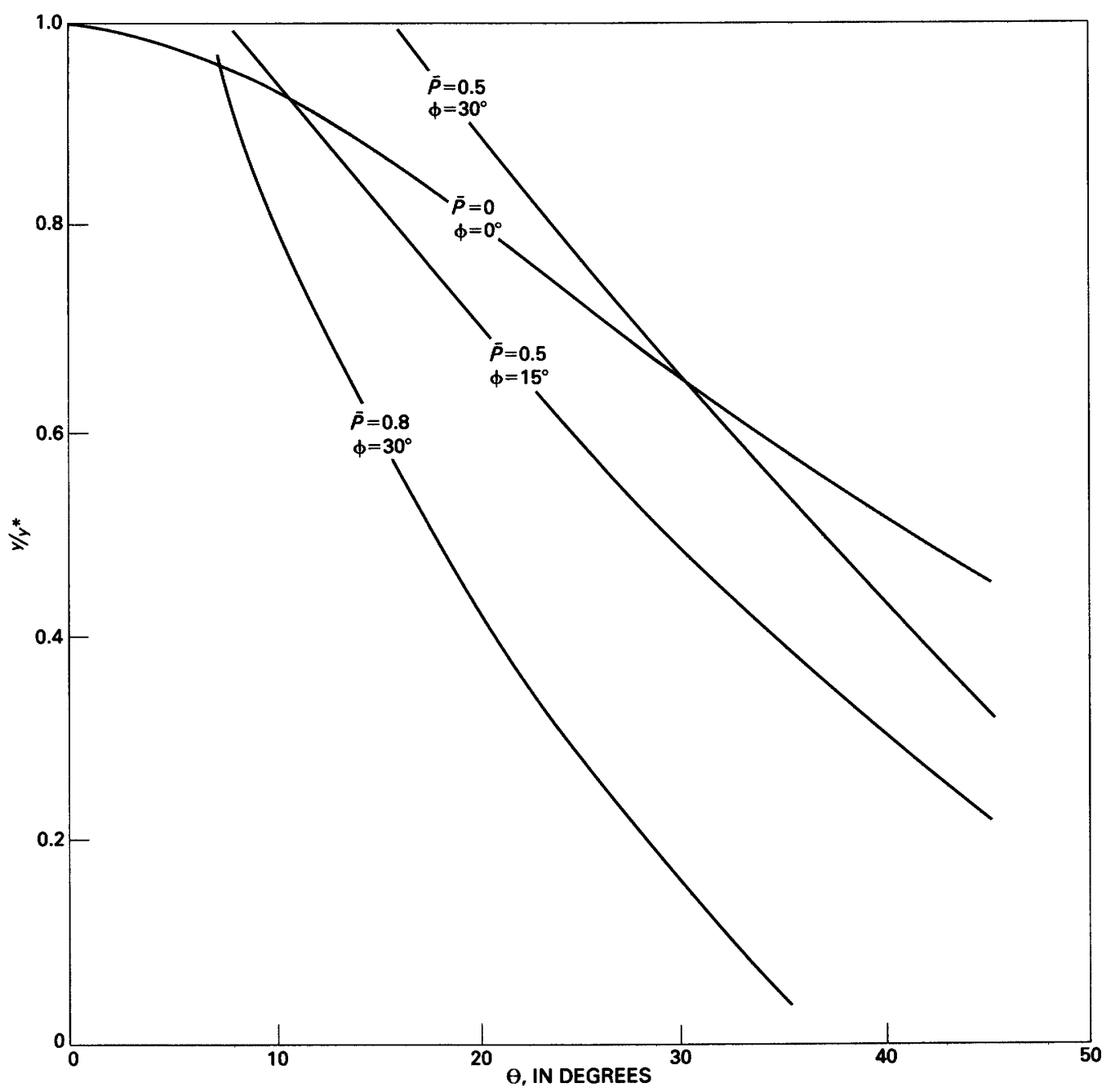

FIGURE 12.-Graph showing dimensionless depth $y / y^{*}$ of maximum values of $\bar{\sigma}_{x}^{\prime}$ in compressive flow as a function of $\theta$ for various values of $\phi$ and $\bar{P}$.

\section{DETERMINATION OF THE VELOCITY FIELD}

To determine the velocity field in our idealized landslide, we use equations $(32 a, b, c)$ for the deformation rates, equation (36) for continuity, and equation (37) for isotropy. We assume that $v$, the vertical velocity, is independent of $x$. Such an assumption is reasonable for an infinitely long slope. Then, from equation (36) for continuity we have

$$
\frac{\partial^{2} v}{\partial x \partial y}=0
$$

and

$$
\frac{\partial^{2} u}{\partial x^{2}}=0
$$

or

$$
u=r x+F(y)+u_{\mathrm{o}},
$$

where $r$ and $u_{\mathrm{o}}$ are constants and

$$
\frac{\partial u}{\partial x}=r
$$

Note that in equation (108) $r$ represents a constant longitudinal deformation rate, or from equation (32a) that

$$
\dot{e}_{x}=-\frac{\partial u}{\partial x}=-r
$$

Under these conditions, equation (36) for continuity becomes 


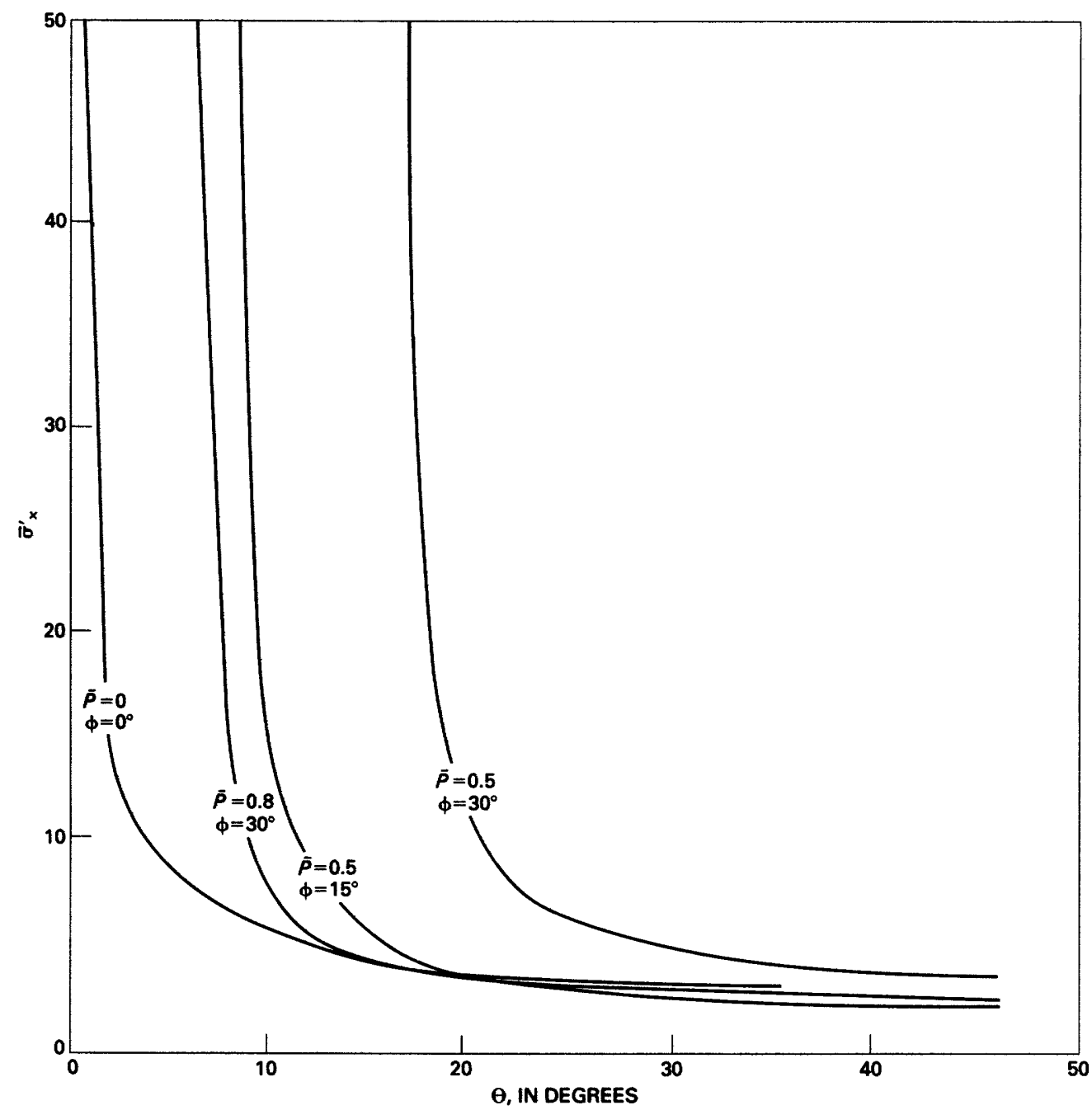

FIGURE 13.-Graph showing maximum value of $\bar{\sigma}_{x}^{\prime}$ in compressive flow as a function of $\theta$ for various values of $\phi$ and $\bar{P}$.

$$
\frac{\partial v}{\partial y}=r\left[\frac{\sin \phi+\cos 2 \alpha}{\sin \phi-\cos 2 \alpha}\right]
$$

and equation (37) for isotropy becomes

$$
\frac{\partial u}{\partial y}=\left[\frac{\partial u}{\partial x}-\frac{\partial v}{\partial y}\right] \tan 2 \alpha
$$

Substituting equations (108) and (110) in equation (111), we arrive at

$$
\frac{\partial u}{\partial y}=\frac{-2 r \sin 2 \alpha}{\sin \phi-\cos 2 \alpha}=F^{\prime}(y) .
$$

Since $\alpha=45^{\circ}-\frac{\phi}{2}$ on $y=y^{*}$, we see that infinities occur in $\frac{\partial v}{\partial y}$ (equation 110) and $\frac{\partial u}{\partial y}$ (equation 112) on the basal slip plane of the landslide. Such infinities occur because of rapid velocity changes across a narrow zone on the base of a landslide.

The discontinuity in the vertical deformation rate $\frac{\partial v}{\partial y}$ relates to the volume dilatancy rate. A nonzero volume dilatancy rate occurs if the soil making up the landslide is frictional-that is, if $\phi \neq 0$. Expressions for volume dilatancy rates in compressive and extending flow are easily derived from equation (33) for the sum of principal deformation rates, equation (108) for $\frac{\partial u}{\partial x}$, and equation (110) for $\frac{\partial v}{\partial y}$. Combining these equations, we obtain the dilatancy rate

$$
\dot{d}=\frac{2 r \sin \phi}{\cos 2 \alpha-\sin \phi}
$$

as a function of $\alpha$, the angle that $\sigma_{1}^{\prime}$ makes with the 
A

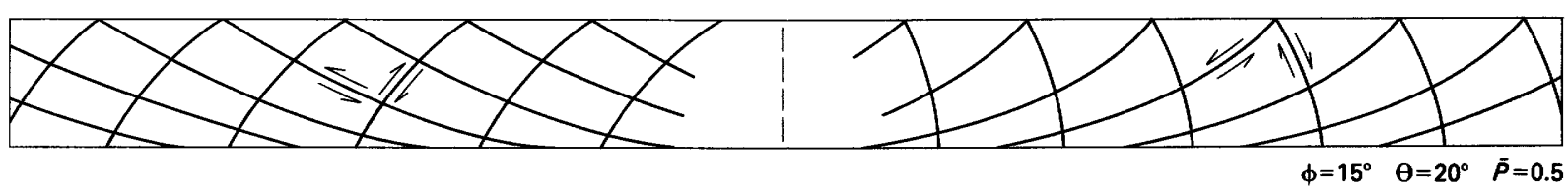

B

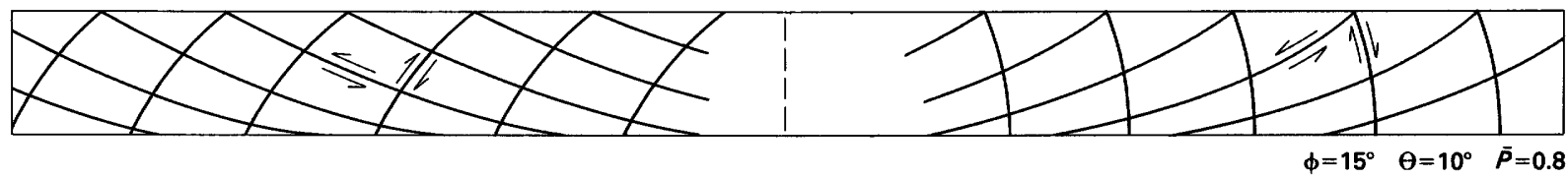

c

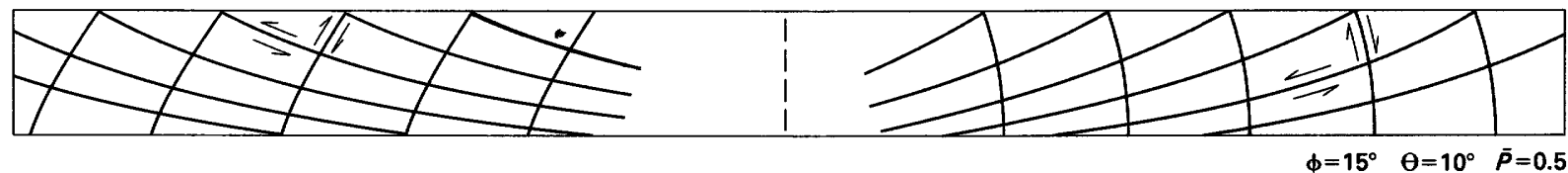

D

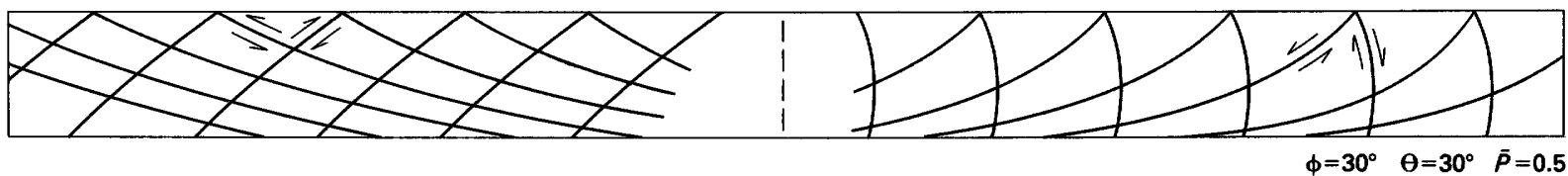

E
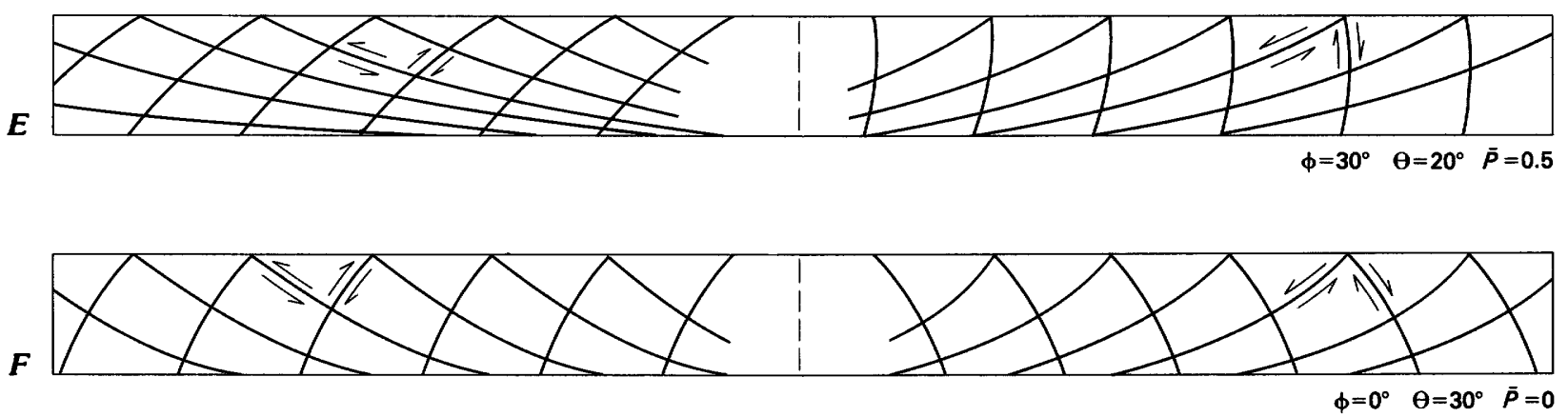

FIGURE 14.-Sketches showing stress characteristics for various slope angles $\theta$, angles of internal friction $\phi$, and dimensionless pore pressures $\bar{P}$. Values of $\theta, \phi$, and $\bar{P}$ used for each case are given below the lower right-hand corner of each sketch. Arrows indicate the relative sense of slip across the characteristics and the dashed lines separate regions of compressive and extending flow.

positive $x$ axis. Here, $r$ is negative for compressive flow and positive for extending flow. Using equation (78) the volume dilatancy rate $\dot{d}$ can be obtained as a function of $y / y^{*}$. The results are shown in figures $15 A$ and $15 B$ for some values of $\theta, \phi$, and $\bar{P}$. Note that the negative sign on $\dot{d}$ represents volume expansion. The dilatancy rate in every case where $\phi$ is nonzero is infinite on the landslide base because of the discontinuity in $\frac{\partial v}{\partial y}$ on $y=y^{*}$. When $y=0$, the dilatancy rate increases with $\phi$ for extending and compressive flow. In general, the dilatancy rate is greater at a given depth in compressive flow than in extending flow. The dilatancy rate increases as $\phi$ increases or as $\bar{P}$ or $\theta$ decreases-a consequence of increasing shear stress in each of these cases. Finally, as we can see from equation (113), the dilatancy rate vanishes when $\phi=0$; thus flow in the purely cohesive case is incompressible.

Returning to equations (110) and (112), we next integrate these equations to find the $x$ and $y$ components of velocity, $u$ and $v$, of the landslide as functions of $\alpha$. Since $v$ is a function of $y$ only, from equation (110) for $\frac{\partial v}{\partial y}$ we have 


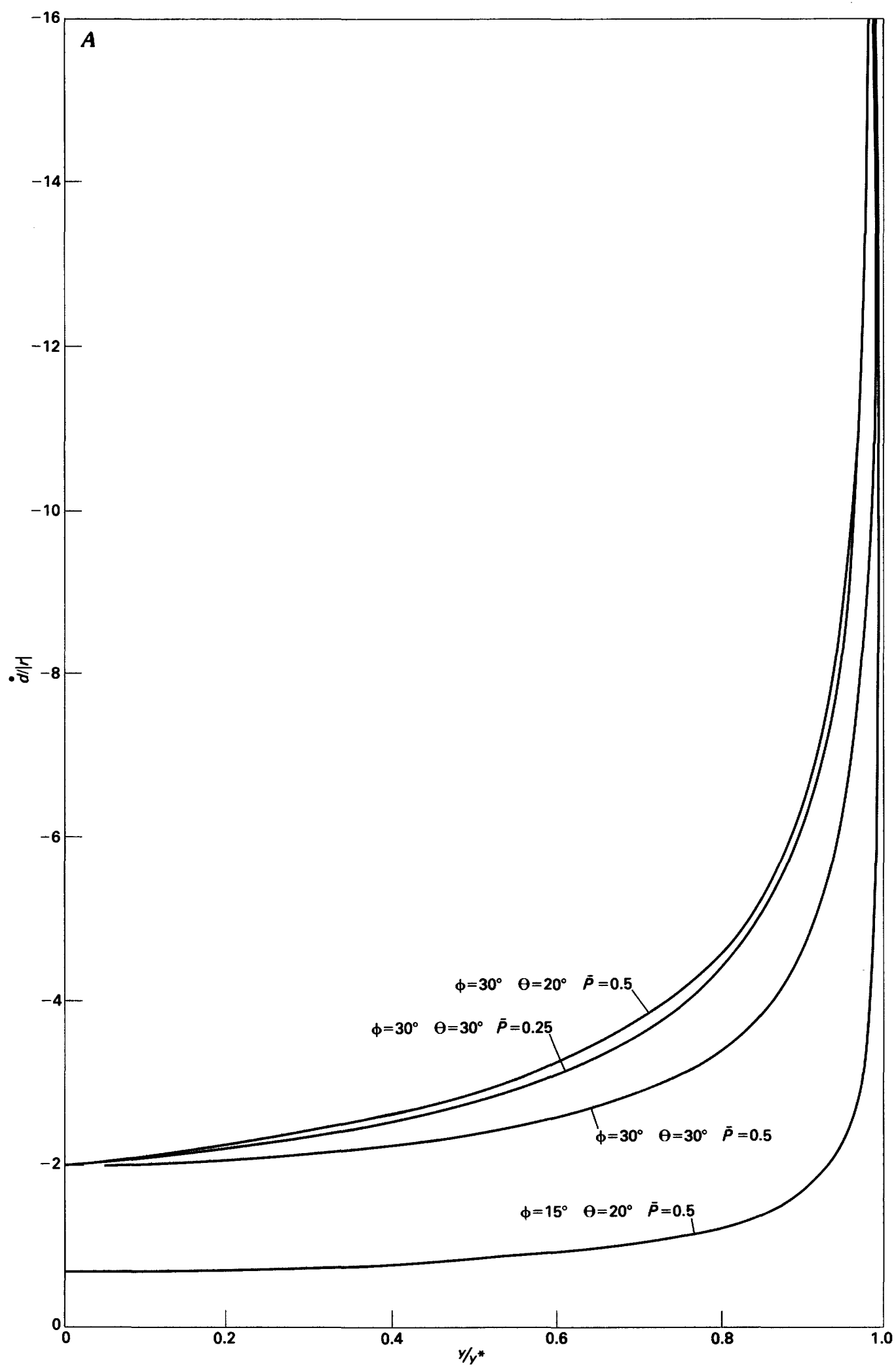

FIGURE 15 (above and facing page).-Graphs showing variation of dimensionless dilatancy rate $d \dot{d}|| r \mid$ with dimensionless depth $y / y^{*}$ for various values of $\theta, \phi$, and $\bar{P}$. $A$, Compressive flow. $B$, Extending flow. 


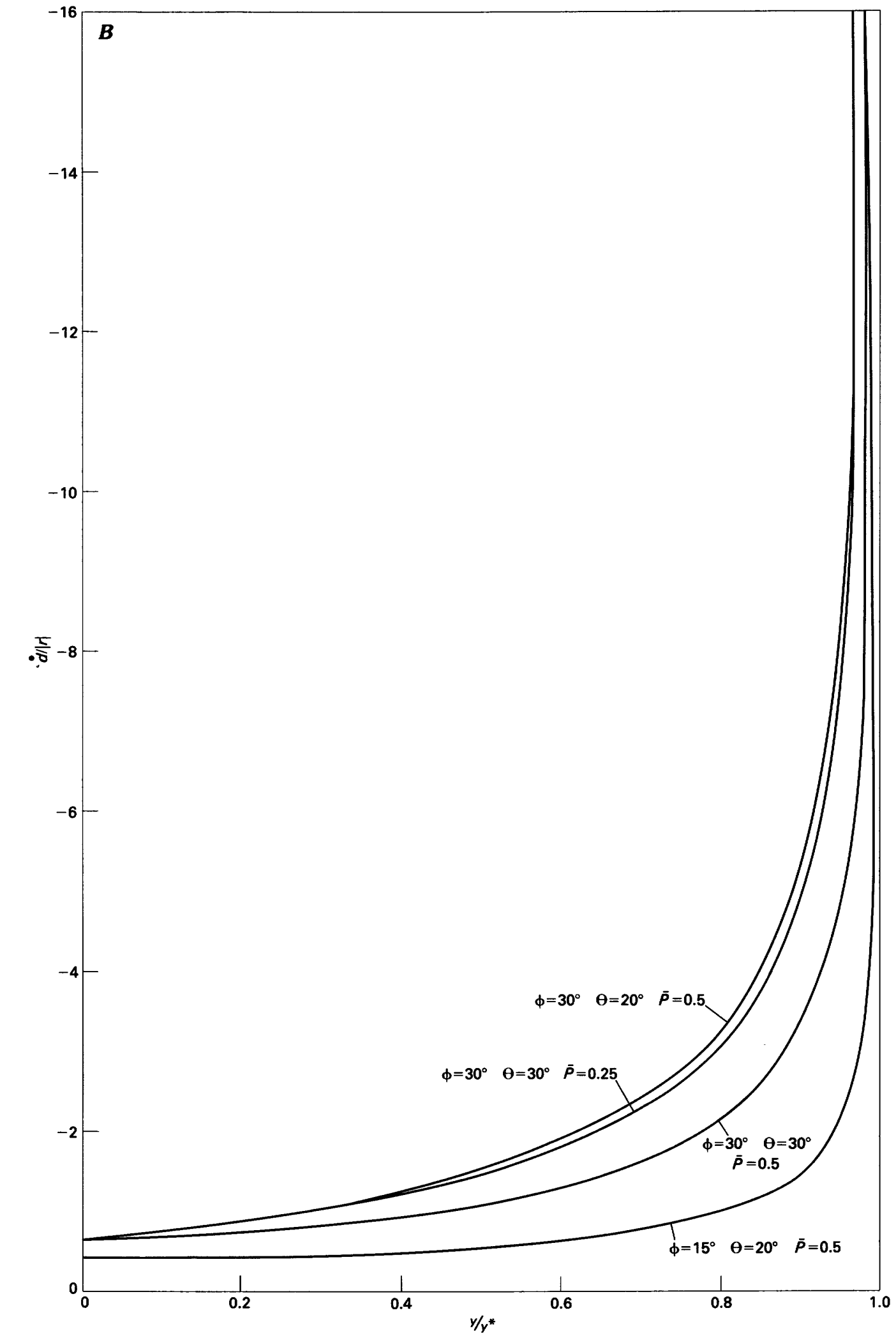




$$
\begin{gathered}
v=r \int\left[\frac{\sin \phi+\cos 2 \alpha}{\sin \phi-\cos 2 \alpha}\right] d y+B \\
=2 y^{*} r[\sin \delta+\bar{P} \sin \phi] \sin \theta \\
\int \frac{[\sin \phi+\cos 2 \alpha] d \alpha}{[\sin \theta-\sin \phi(\sin 2 \alpha \cos \theta+\cos 2 \alpha \sin \phi)} \\
+\bar{P} \sin \phi \sin 2 \alpha]^{2} \\
+B, \quad(114)
\end{gathered}
$$

where the differential of equation (78) with respect to $\alpha$ replaces $d y$. Then, by integrating equation (114) (Gradshteyn and Ryzhik, 1980, p. 149), we get

$$
\begin{aligned}
\frac{v}{y^{*} r}= & -[\sin \delta+\bar{P} \sin \phi] \sin \theta\left\{F_{1}[\phi, \theta, \bar{P}]\right. \\
& \left.+G_{1}[\phi, \theta, \bar{P}]\right\}+B,
\end{aligned}
$$

where

$$
F_{1}[\phi, \theta, \bar{P}]=
$$

$\sin \phi(\bar{P}-\cos \theta)(1+\sin \phi \cos 2 \alpha)$

\begin{tabular}{c}
$+\sin \theta\left(1+\sin ^{2} \phi\right) \sin 2 \alpha$ \\
\hline$D_{2}[\sin \theta-\sin \phi \sin \theta \cos 2 \alpha$ \\
$+\sin \phi(\bar{P}-\cos \theta) \sin 2 \alpha]$
\end{tabular}

and

$$
G_{1}[\phi, \theta, \bar{P}]=\frac{4 \sin \phi \sin \theta}{\left(D_{2}\right)^{3 / 2}}
$$

$\tan ^{-1}\left[\frac{\sin \theta(1+\sin \phi) \tan \alpha+\sin \phi(\bar{P}-\cos \theta)}{\left(D_{2}\right)^{1 / 2}}\right]$

Equation (115) is subject to the condition given by relation (101), and since $v=0$ when $y=y^{*}$, we have

$$
\begin{gathered}
B=\frac{(\sin \delta+\bar{P} \sin \phi)^{2} \sin \theta\left(1+\sin ^{2} \phi\right)}{D_{2}(\sin \theta-\bar{P} \sin \phi \cos \phi-\sin \phi \cos \delta)} \\
+\frac{4(\sin \delta+\bar{P} \sin \phi) \sin \phi \sin ^{2} \theta}{\left(D_{2}\right)^{3 / 2}} \\
\tan ^{-1} \frac{\sin \delta+\bar{P} \sin \phi}{\left(D_{2}\right)^{1 / 2}}
\end{gathered}
$$

By following a similar procedure for equation (112), we find that $\begin{aligned} \frac{u-u_{\mathrm{o}}}{y^{*} r} & =[\sin \delta+\bar{P} \sin \phi] \sin \theta\left[F_{2}[\phi, \theta, \bar{P}]\right. \\ & \left.+G_{2}[\phi, \theta, \bar{P}]\right]+C,\end{aligned}$

where

$$
F_{2}[\phi, \theta, \bar{P}]=\frac{2 \sin \theta(\sin \phi-\cos 2 \alpha)}{\begin{array}{c}
D_{2}[\sin \theta-\sin \phi \sin \theta \cos 2 \alpha \\
+\sin \phi(\bar{P}-\cos \theta) \sin 2 \alpha]
\end{array}}
$$

and

$$
G_{2}[\phi, \theta, \bar{P}]=\frac{4 \sin \phi(\bar{P}-\cos \theta)}{\left(D_{2}\right)^{3 / 2}}
$$

$\tan ^{-1}\left[\frac{\sin \theta(1+\sin \theta) \tan \alpha+\sin \phi(\bar{P}-\cos \theta)}{\left(D_{2}\right)^{1 / 2}}\right]$.

Equation (119) is also subject to the condition given by relation (101), and since we are only interested in how $u$ varies with $\alpha$ on an arbitrary cross section, we ignore the term in $x$ given in equation (107). The term $u_{\mathrm{o}}$ represents the sliding velocity when $y=y^{*}$. For $u$ to equal $u_{\mathrm{o}}$ when $y=y^{*}$, we must have

$$
\begin{gathered}
C=\frac{4(\sin \delta+\bar{P} \sin \phi) \sin \theta \sin \phi(\bar{P}-\cos \theta)}{\left(D_{2}\right)^{3 / 2}} \\
\tan ^{-1}\left[\frac{\sin \delta+\bar{P} \sin \phi}{\left(D_{2}\right)^{1 / 2}}\right] .
\end{gathered}
$$

When $\phi=0$, equations (115) and (119) for the velocities reduce to

$$
\frac{v}{y^{*} r}=1-\sin 2 \alpha=1-\frac{y}{y^{*}}
$$

and

$$
\frac{u-u_{\mathrm{o}}}{y^{*} r}=-\cos 2 \alpha=\mp 2\left[1-\left(y / y^{*}\right)^{2}\right]^{1 / 2} .
$$

Here, the relationships $y / y^{*}=\sin 2 \alpha$ and $\cos 2 \alpha=$ $\pm\left[1-\left(y / y^{*}\right)^{2}\right]^{1 / 2}$, which apply when $\phi=0$, have been invoked. These expressions are, with notational and coordinate axis differences, the same as those presented by Nye (1951, equation 9, p. 559) for velocities in an alpine glacier.

The dimensionless velocities $\left[\left(u-u_{0}\right) / r y^{*}\right]$ and $v / r y^{*}$ are shown as functions of $y / y^{*}$ (where $y / y^{*}$ has been obtained from equation (78)) for compressive and extending flow and various values of $\phi, \theta$, and $\bar{P}$ in figure 16. 

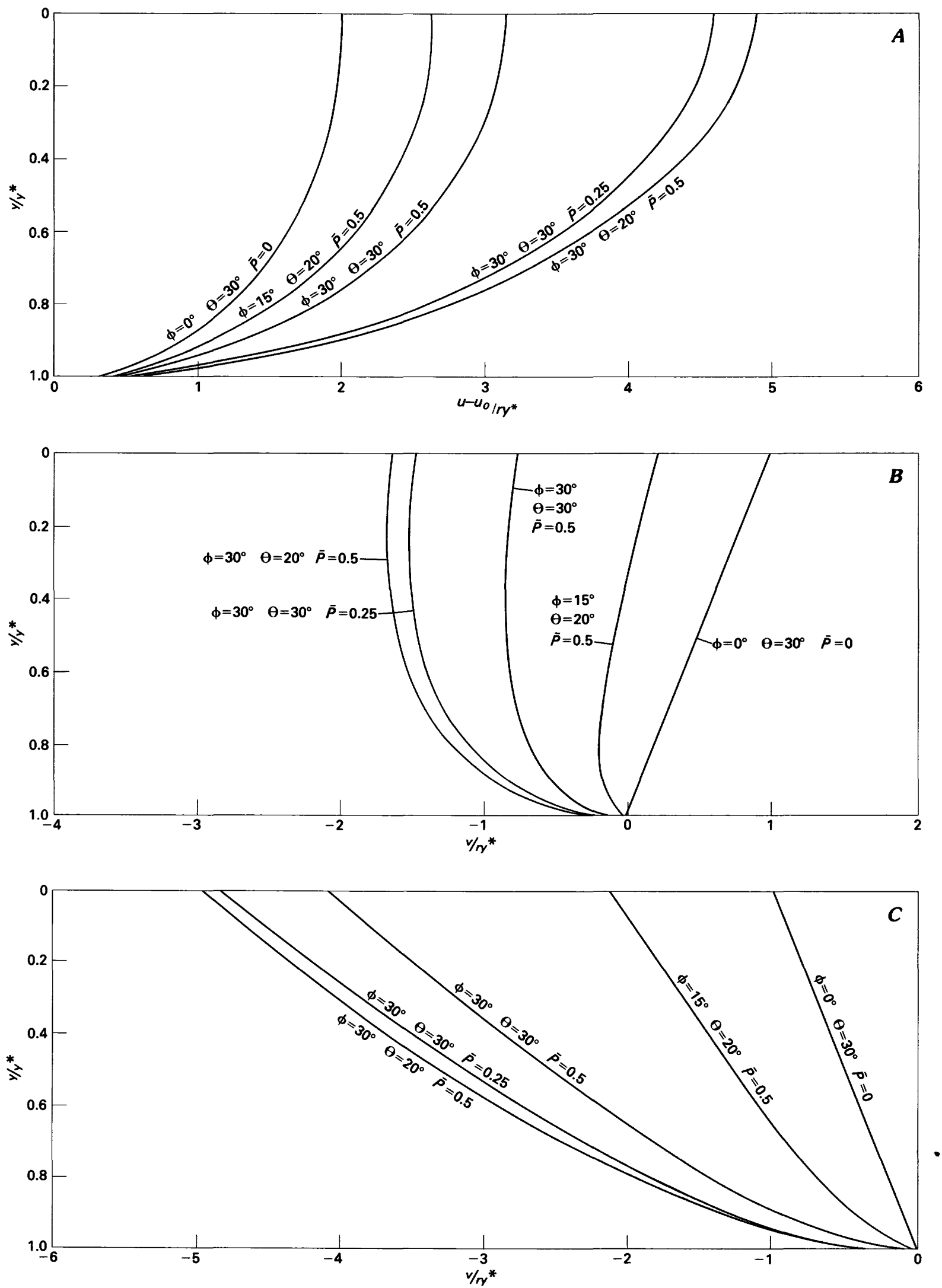

FIGURE 16.-Graphs showing dimensionless velocities $\left(u-u_{0}\right) / r y^{*}$ and $v / r y^{*}$ as functions of dimensionless depth $y / y^{*}$ for compressive and extending flow and various values of $\theta, \phi$, and $\bar{P}$. $A$, Dimensionless longitudinal velocity for compressive and extending flow. $B$, Dimensionless normal velocity for extending flow. $C$, Dimensionless normal velocity for compressive flow. 
Figure 16 shows that the dimensionless longitudinal velocity $\left[\left(u-u_{0}\right) / r y^{*}\right]$ is the same in compressive and extending flow. This velocity decreases as $\theta$ increases and as $\phi$ decreases. The dimensionless longitudinal velocity also decreases as $\bar{P}$ increases. Note that, in the purely cohesive case, the velocities are independent of $\bar{P}$, because $y^{*}$ in equations (123) and (124) is independent of $\bar{P}$ when $\phi=0$. (See equation (74).) These results for $\left(u-u_{0}\right) / r y^{*}$ are consistent with the fact that the allowable shear stress, and hence the shear strain rate (equation (112)) within the slide, is increased as $\phi$ is increased or as $\bar{P}$ and $\theta$ are reduced. Finally, although the observation that the dimensionless longitudinal velocity decreases with increasing slope angle seems contradictory, recall that $\left(u-u_{0}\right) / r y^{*}$ given by equation (119) occurs in response to deformation within the landslide mass and this velocity is relative to the basal slide velocity $u_{0}$, which increases with slope angle.

Since the dimensionless $y$ component of velocity $v / r y^{*}$ is positive in the positive direction of the $y$ axis (fig. 3 ), $v / r y *$ is more negative in compressive than in extending flow as figure 16 shows. We see a similar effect as $\theta$ is reduced and as $\phi$ is increased. Again, reducing $\bar{P}$ causes a more negative $v / r y^{*}$. Whether the flow is compressive or extending, a more negative value of $v$ means a greater dilatancy rate in the landslide. This result is consistent with the observation made previously that increasing $\phi$, and decreasing $\bar{P}$ or $\theta$, increases the shear stress and enhances the dilatancy rate as seen in figure 15 .
So far, we have discussed the velocity distribution within the idealized landslide for extending and compressive flow. There is a third case where $r$, the longitudinal deformation rate in equation (108), vanishes. When $r$ vanishes the longitudinal velocity $u$ given by equation (119) decreases to the basal sliding velocity $u_{o}$, and the $y$ component of velocity $v$ given by equation (115) vanishes. The landslide then slides as a rigid body on the basal slip plane, and the flow is termed plug flow (Nye, 1951).

As mentioned earlier, the velocity and stress characteristics for our Coulomb material coincide. Thus, potential discontinuities in velocities and strain rates will be propagated along the stress characteristics (slip surfaces) shown in figure 14. Also, we found that discontinuities in velocity originating at, say, the line of discontinuity $y=y^{*}$ will be propagated in an exponential manner with change in the angle $\alpha$ along the slip lines (equations (52), (53), (61), and (62)).

Suppose a local irregularity at a point $Q$ (fig. 17A) on the basal slip plane of a landslide $\left(y=y^{*}\right)$ causes a discontinuity in tangential velocity $\bar{u}_{1}$. This, in turn, propagates a discontinuity of tangential velocity $\bar{u}_{1}$ and a discontinuity of normal velocity $\bar{v}_{1}$ along a $\beta_{\text {_ }}$ characteristic with the sense of relative velocity shown in figure $17 \mathrm{~A}$. The magnitude of the tangential velocity at a point on $\beta_{-}$is given by equation (52) and the magnitude of normal velocity is given by equation (59).
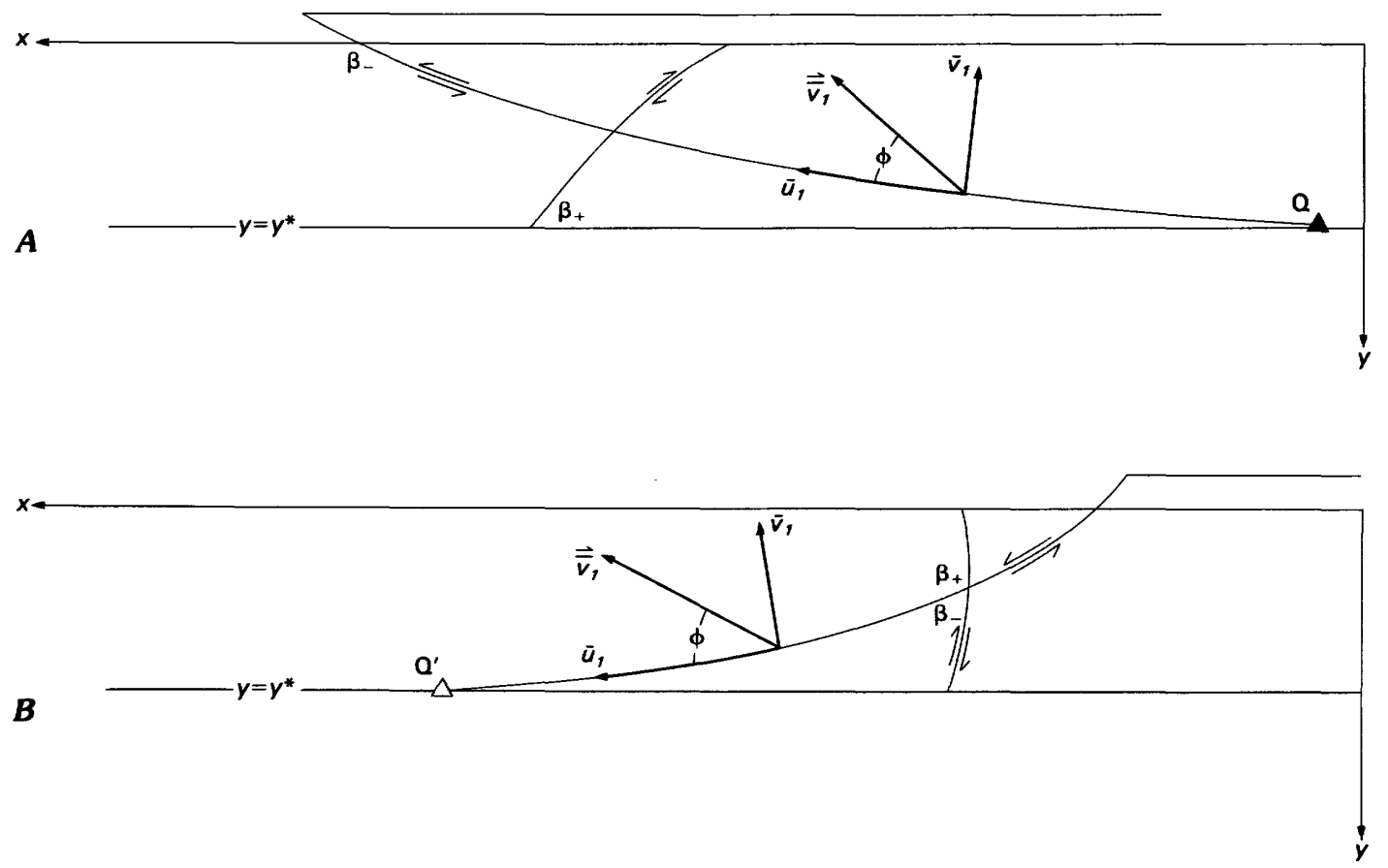

FIGURE 17.-Sketches showing fault surfaces produced by propagation of discontinuities along $\beta_{-}$slip lines in $A$, compressive flow and $B$, extending flow. The directions of the velocity jump components and the resultant velocity jump vectors are also shown. Arrows show sense of relative slip across the fault surfaces. 
Since $\alpha_{o}$ in this case is $45^{\circ}-\frac{\phi}{2}$, the magnitude of the tangential velocity jump on the surface in compressive flow (where $\alpha=0$ ) is given in dimensionless form as

$$
\frac{\bar{u}_{1}}{\bar{u}_{1}}=\exp \left[\left(\frac{\pi}{4}-\frac{\phi}{2}\right) \tan \phi\right],
$$

where $\phi$ is in radians, and the normal velocity jump when $y=0$ is given in dimensionless form as

$$
\frac{\bar{v}_{1}}{\bar{u}_{1}}=-\frac{\bar{u}_{1}}{\bar{u}_{1_{0}}} \tan \phi .
$$

The negative sign indicates that this component acts

upwards. As mentioned earlier, the resultant velocity $\mid$ where $\phi$ is in radians, and the dimensionless magnitude

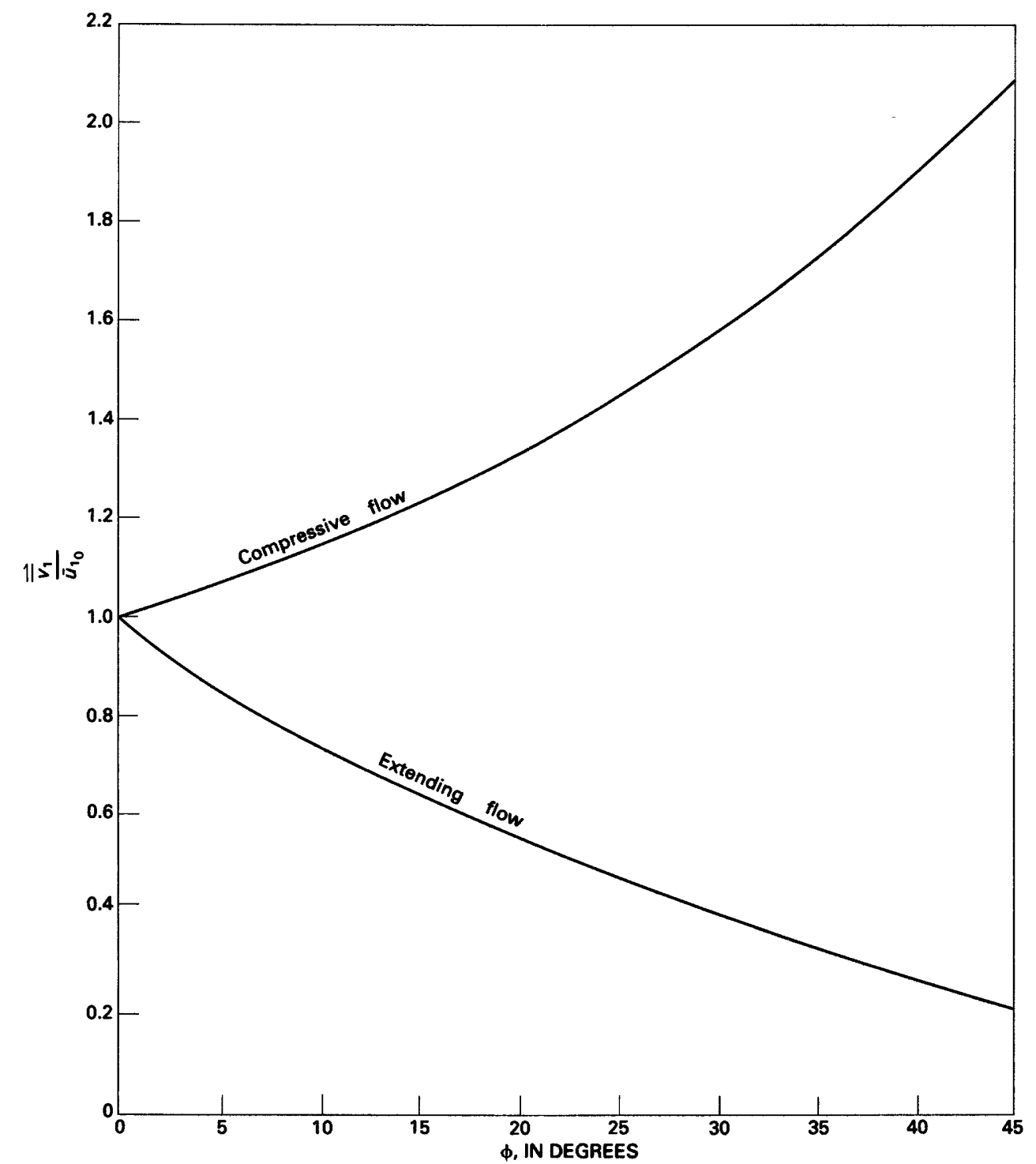

FIGURE 18.-Graph showing magnitude of resultant velocity jump on the surface as a function of angle of internal friction $\phi$ for extending and compressive flow.

jump vector $\overrightarrow{\vec{v}}_{1}$ is inclined at an angle $\phi$ to the $\beta_{-}$slip line, and its magnitude is an increasing function of $\phi$ as seen in figure 18.

Similarly, if basal slip is impeded at a point $Q^{\prime}$ (fig. 17B) when $y=y^{*}$ during extending flow, discontinuities in $\bar{u}_{1}$ and $\bar{v}_{1}$ will propagate along a $\beta_{-}$ characteristic with the sense of relative velocity shown in figure $17 B$. The dimensionless magnitude of the tangential velocity jump $\bar{u}_{1}$ on $y=0$ is given here $(\alpha=$ $90^{\circ}$ when $y=0$ ) by

$$
\frac{\bar{u}_{1}}{\bar{u}_{1}}=\exp \left[-\left(\frac{\pi}{2}-\frac{\phi}{2}\right) \tan \phi\right],
$$


of the normal velocity jump $\vec{v}_{1}$ when $y=0$ is given by equation (126). Again, $\bar{v}_{1}$ is directed toward the ground surface. The resultant velocity jump vector $\overrightarrow{\vec{v}}_{1}$ is inclined at an angle $\phi$ to the $\beta$. slip line, and its magnitude is a decreasing function of $\phi$ (fig. 18).

Physically, the jump in the tangential component of velocity on the surface leads to a thrust fault scarp in compressive flow and a normal fault scarp in extending flow (fig. 18). In compressive flow, the height of the scarp increases as $\phi$ increases; the opposite is true in extending flow. When $\phi=0$, the scarp height is equal to the magnitude of the discontinuity on the base of the landslide, in both compressive and extending flow.

The discontinuity in the normal component of velocity leads to a zone of disturbance along a $\beta_{-}$slip line that is proportional in thickness to the normal velocity discontinuity. Physically, one might expect a disordering of the particle-packing arrangement in this zone-that is, dilatancy. In compressive flow, the thickness of the dilatant zone increases with $\phi$. For extending flow, the thickness of the dilatant zone increases with $\phi$ to a maximum at $\phi \approx 37^{\circ}$, thereafter, decreasing in thickness with increasing internal friction. When $\phi=0$, the normal velocity discontinuity vanishes, and no dilatancy accompanies the propagation of velocity discontinuities on the slip lines. Nye (1951) discussed the propagation of discontinuities in alpine glacier flow-that is, in the purely cohesive case.

\section{DISCUSSION}

\section{SIMILARITIES AND DIFFERENCES BETWEEN NYE'S ALPINE GLACIER MODEL AND OUR LANDSLIDE MODEL}

The flow of landslides is similar to that of alpine glaciers. These similarities occur because soils within landslides can be described as Coulomb plastic materials, a generalization of Mises plastic materials. Like Nye's (1951) Mises plastic model for alpine glaciers, our model for landslides predicts, depending on the longitudinal deformation rate, that a landslide can undergo compressive, extending, or plug flow. For both models, shear and normal stresses increase linearly with depth, and longitudinal stresses increase in an elliptic manner with depth. Both models predict tensile longitudinal stresses and normal fault scarps at the surface in extending flow, and thrust surfaces and thrust fault scarps at the surface in compressive flow. Longitudinal velocities caused by internal deformation in both models are greatest near the surface and decrease elliptically to the basal slip plane where they are equal to the basal slide velocity. Also, like Nye's glacier-flow model, our model predicts that in plug flow a landslide moves as a rigid body at the basal slide velocity. Finally, in both models, the thickness of the plastically flowing region is controlled by the slope angle, the density, and the cohesion. This thickness increases with increasing cohesion and decreases with increasing density and slope angle.

However, the landslide model and glacier model are in some ways dissimilar. For example, unlike the alpine glacier model, our model is affected by pore pressure and internal friction as well as slope angle, density, and cohesion. Increasing the pore pressure or decreasing the angle of internal friction for a given slope angle and cohesion decreases the thickness of the plastically flowing region.

The models also differ because stresses in the landslide are influenced by pore pressure and internal friction. In extending flow, all stresses are smaller and the depth to which longitudinal stresses are tensile is greater when pore pressure is increased or when internal friction is decreased for a given slope angle, density, and cohesion.

Another difference is that the stress characteristics are influenced by pore pressure and internal friction in the landslide model. They flatten as $\phi$ is increased or $\bar{P}$ is decreased because of the increase in depth of the basal slide plane with increasing internal friction or decreasing pore pressure. An excess pore pressure also leads to a bounded slip line field even where the slope angle is less than the angle of internal friction, a point made by Crans and Mandl (1981).

As in Nye's (1951) model for alpine glacier flow, longitudinal velocities occurring in response to deformation in the landslide are the same in both extending and compressive flow. However, in the landslide model, internal deformation and hence longitudinal velocities increase as the pore pressure is reduced or as internal friction is increased. Also, in both models the sense of the $y$ component of velocity is consistent with vertical expansion in compressive flow and vertical contraction in extending flow. But, in the landslide model, the vertical expansion in compressive flow is amplified by dilatancy and the vertical contraction in extending flow is reduced by dilatancy. Dilatancy vanishes for $\phi=0$.

Dilatancy also influences the propagation of velocity discontinuities in the landslide model as the resultant velocity jump vector is inclined at the angle of internal friction to the slip lines. As we have noted, the normal component of velocity discontinuity vanishes when $\phi=0$. 


\section{THE ASSUMPTION OF ASSOCIATED FLOW}

The dilatancy predicted by our model is a consequence of assuming an associated flow rule. Such a flow rule assumes that at any smooth point on a yield surface the plastic strain rate vector is normal to the yield surface (Drucker, 1956; Davis, 1968). The advantages of this assumption are (1) velocity and stress characteristics coincide, and (2) simple and useful limit theorems apply only to such materials (Davis, 1968). The principal disadvantage of assuming associated flow is that it leads to an unrealistically large dilatancy for Coulomb plastic materials, exemplified by the occurrence of infinite dilatancy rates on the basal slip plane in our landslide model (fig. 15).

The implication that Coulomb plastic soils expand indefinitely and yet retain shear strength is physically implausible, and in fact, no known material behaves in this way (Houlsby and Wroth, 1980). Houlsby and Wroth (1980) pointed out that soils, when sheared monotonically, first dilate and then approach a critical state in which deformation occurs at constant volume.

An alternative (Davis, 1968; Salencon, 1977; Houlsby and Wroth, 1980), then, is to replace the angle of internal friction in equations $(32 a, b, c)$ with a variable angle of dilation, $\nu$, which when deformation begins, equals the angle of internal friction $\phi$, and with continued deformation drops to zero. Theoretically, the angle $\nu$ could be larger than $\phi$. However, this would imply volumetric expansion under hydrostatic compression.

Using a variable angle of dilation would add considerable complexity to the analysis unless we assuine that the deformation being modeled has progressed to the point where $\nu=0$. Since $\phi \neq \nu$, the flow rule would be nonassociated-that is, stress and velocity characteristics would no longer be coincident, and the simple limit theorems referred to above would no longer apply.
In any case, velocity characteristics initially intersecting at an angle of $90^{\circ}-\phi$ would, in the course of continued flow, come to intersect at $90^{\circ}$ and would be equivalent to the $\phi=0$ examples given earlier for the landslide model. Thus velocities, velocity characteristics, and velocity jump relations given for $\phi=0$ in the landslide model apply when the angle of dilation $\nu$ is zero.

Another approach to the initiation and growth of slip surfaces is the Rice (1973) and Rudnicki and Rice (1975) proposal that the physical mechanisms involved in dilatant materials lead to vertices on the yield surfaces and consequently to nonassociated flow rules. Flows described by such flow rules are unstable and lead to localized planar zones of nonuniform deformation in otherwise homogeneously deforming materials. Of course, resolving the issue of whether an associated or nonassociated flow rule applies in a given landslide depends on careful field and laboratory observations.

\section{CONGLUDING REMARKS}

The model for landslide flow presented here is by no means a complete solution. Landslides are complicated by variable geology and topography, factors obviously missing in our idealized isotropic, homogeneous, inclined half-space. However, like Nye (1951), we have simplified our model so that some essential features of the mechanics of landslide flow can be highlighted. Any field test of our model should be carried out under conditions that best approximate our assumptions. Such field tests would be best carried out in relatively homogeneous and isotropic landslides that are long and shallow, and in which regions of compressive, extending, and plug flow are clearly identified. An idealized sketch of such a field situation is shown in figure 19.

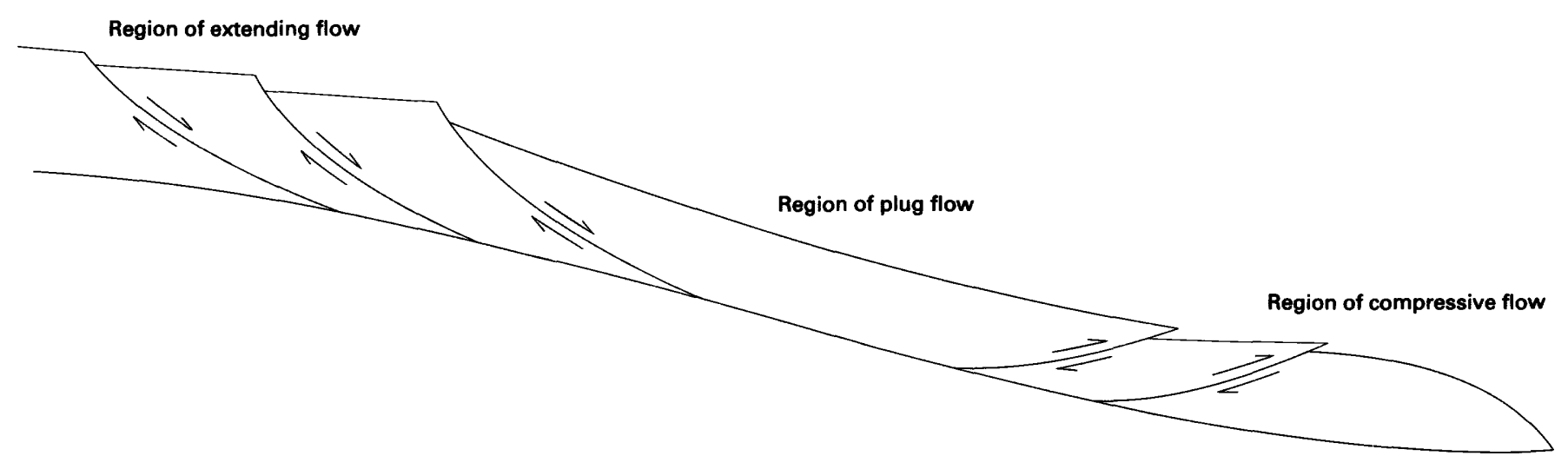

FIGURE 19.-Sketch of an idealized long, shallow landslide. Arrows indicate relative senses of slip along failure surfaces in extending and compressive flow. 


\section{REFERENCES CITED}

Abbott, M. B., 1966, An introduction to the method of characteristics: New York, Elsevier, 243 p.

Coulomb, C. A., 1773 [1776], Essai sur une application des règles de maximis \& minimis à quelques problèmes de statique, relatifs à l'architecture: Memoires de Mathématique \& de physique, présentés à l'Académie Royale des Sciences (Savants Étrang.), v. 7, p. 343-382. [Reprinted and translated in Heyman, Jacques, 1972, Coulomb's memoir on statics: London, Cambridge University Press, 212 p.]

Crandall, S. H., 1956, Engineering analysis: New York, McGraw-Hill, $417 \mathrm{p}$.

Crans, W., and Mandl, G., 1981, On the theory of growth faulting, Pt. II(b) Genesis of the "unit": Journal of Petroleum Geology, v. 3 , no. 3 , p. $333-355$.

Davis, E. H., 1968, Theories of plasticity and the failure of soil masses, in Lee, I. K., ed., Soil mechanics-Selected topics: New York, Elsevier, p. 341-380.

Drucker, D. C., 1956, On uniqueness in the theory of plasticity: Quarterly of Applied Mathematics, v. 14, no. 1, p. 35-42.

Drucker, D. C., and Prager, W., 1952, Soil mechanics and plastic analysis or limit design: Quarterly of Applied Mathematics, v. 10, no. 2 , p. $157-165$.

Frontard, M., 1922, Cycloides de glissement des terres: Academy of Sciences [Paris], Comptes Rendus, v. 174, p. 526-528.

Gradshteyn, I. S., and Ryzhik, I. M., 1980, Table of integrals, series, and products: New York, Academic Press, 1160 p.

Hill, R., 1950, The mathematical theory of plasticity: London, Oxford University Press, 355 p.

Houlsby, G. T., and Wroth, C. P., 1980, Strain and displacement discontinuities in soil: American Society of Civil Engineers
Proceedings, Journal of the Engineering Mechanics Division, v. 106, no. EM4, p. 753-771.

Johnson, A. M., 1965, A model for debris flow: University Park, Pa., The Pennsylvania State University, Ph. D. dissertation.

Nye, J. F., 1951, The flow of glaciers and ice-sheets as a problem in plasticity: Royal Society [London] Proceedings, ser. A., v. 207, no. 1091 , p. 554-572.

Pariseau, W. G., 1970, Discontinuous velocity fields in gravity flow of granular materials through slots: Powder Technology, v. 3, p. 218-226.

Prager, William, and Hodge, P. E., Jr., 1951, Theory of perfectly plastic solids: New York, Dover, 264 p.

Rice, J. R., 1973, The initiation and growth of shear bands, in Palmer, A. C., ed., Plasticity and soil mechanics: Cambridge, Cambridge University, Engineering Department, p. 263-274.

Rudnicki, J. W., and Rice, J. R., 1975, Conditions for localization of deformation in pressure-sensitive dilatant materials: Journal of the Mechanics and Physics of Solids, v. 23, p. 371-394.

Salencon, J., 1977, Applications of the theory of plasticity in soil - mechanics: New York, John Wiley, 158 p.

Shield, R. T., 1953, Mixed boundary value problems in soil mechanics: Quarterly of Applied Mathematics, v. 11, p. 61-75.

1955 , On Coulomb's law of failure in soils: Journal of the Mechanics and Physics of Solids, v. 4, no. 1, p. 10-16.

Sokolovski(i), V. V., 1960, Statics of soil media: London, Butterworths, $237 \mathrm{p}$. $270 \mathrm{p}$.

1965, Statics of granular media: Oxford, Pergamon Press,

Terzaghi, Karl, 1943, Theoretical soil mechanics: New York, John Wiley, $510 \mathrm{p}$.

Varnes, D. J., 1962, Analysis of plastic deformation according to Von Mises' theory, with application to the South Silverton area, San Juan County, Colorado: U.S. Geological Survey Professional Paper 378-B, 49 p. 